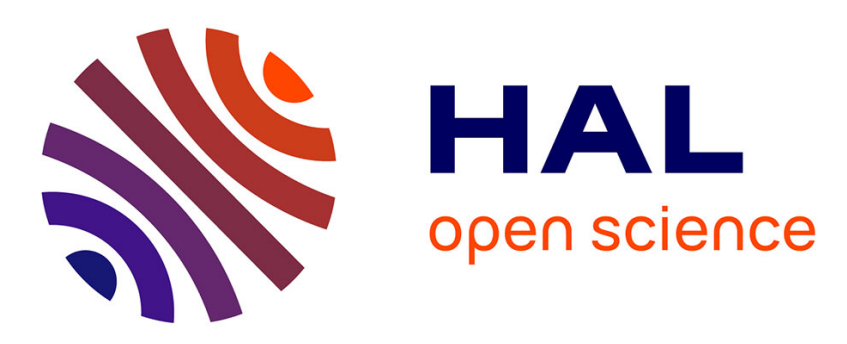

\title{
A generalized Timoshenko beam with embedded rotation discontinuity
}

Ibrahim Bitar, Panagiotis Kotronis, Nathan Benkemoun, Stéphane Grange

\section{To cite this version:}

Ibrahim Bitar, Panagiotis Kotronis, Nathan Benkemoun, Stéphane Grange. A generalized Timoshenko beam with embedded rotation discontinuity. Finite Elements in Analysis and Design, 2018, 150, pp.3450. 10.1016/j.finel.2018.07.002 . hal-01853744

\section{HAL Id: hal-01853744 \\ https://hal.science/hal-01853744}

Submitted on 12 Feb 2019

HAL is a multi-disciplinary open access archive for the deposit and dissemination of scientific research documents, whether they are published or not. The documents may come from teaching and research institutions in France or abroad, or from public or private research centers.
L'archive ouverte pluridisciplinaire $\mathbf{H A L}$, est destinée au dépôt et à la diffusion de documents scientifiques de niveau recherche, publiés ou non, émanant des établissements d'enseignement et de recherche français ou étrangers, des laboratoires publics ou privés. 


\title{
A generalized Timoshenko beam with embedded rotation discontinuity
}

\author{
Ibrahim Bitar $^{\mathrm{a}, *}$, Panagiotis Kotronis ${ }^{\mathrm{a}}$, Nathan Benkemoun ${ }^{\mathrm{c}}$, Stéphane Grange ${ }^{\mathrm{b}}$ \\ a École Centrale de Nantes, Université de Nantes, CNRS, Institut de Recherche en Génie Civil et Mécanique (GeM), UMR 6183, 1 rue de la Noë, BP 92101, 44321, Nantes, cedex 3, France \\ b Univ. Lyon, INSA-Lyon, GEOMAS, F-69621, Villeurbanne cedex, France \\ c IUT Saint-Nazaire, Université de Nantes, CNRS, Institut de Recherche en Génie Civil et Mécanique (GeM), UMR 6183, 58 rue Michel Ange, 44600, Saint-Nazaire, France
}

\begin{abstract}
A novel generalized Timoshenko beam finite element with higher order shape functions is proposed to simulate structural failure. An embedded rotation discontinuity is adopted at the element level to describe the development of plastic hinges and crack opening. The material behaviour at the discontinuity is characterized by a discrete linear law linking the bending moment and the rotational jump, which allows capturing the released fracture energy. Outside the discontinuity, the behaviour is reproduced within the framework of elasto-plastic generalized standard materials. The generalized enhanced Timoshenko beam finite element, the variational formulation and the specific computational procedures are detailed. Numerical examples of a cantilever beam submitted to a rotation, to a transversal displacement and a two-storey reinforced concrete frame illustrate the capacity of the new beam formulation to regularize the results and to reproduce the structural behaviour up to failure.
\end{abstract}

\section{Introduction}

In order to realistically simulate structural behaviour up to failure, one should be able to reproduce strain localization and the opening of cracks. Strain localization occurs prior to rupture for different type of materials: granular materials [1,2], quasi-fragile materials such as concrete $[3,4]$ and rocks $[5,6]$, materials with an elasto-plastic behaviour with softening [7].

A classical approach to correctly simulate this phenomenon is to add explicitly or implicitly a length scale parameter on the continuum mechanics model by considering a spatial dependency in the constitutive equations [8], generally via an integral over space [9] or in a gradient form [10-12]. The recent Thick Level-Set (TLS) model [13] is a thermodynamically sound approach with a non constant internal length and a smooth transition from damage to fracture. From a mathematical point of view, the absence of a characteristic length leads to a poorly posed problem; the hyperbolic character of the governing equations is lost [14], causing pathological dependence of the results on the finite element mesh. Numerical analyses showed that fracture is ultimately done without dissipation of energy [15].
Another method, traced back to the work of the Cosserat brothers in the early $\mathrm{XX}^{\text {ieth }}$ century [16-20], is to use continua with microstructure. They are kinematically enriched models, whether micromorphic or of the higher order gradient type [21], with additional kinematic description that implicitly introduces a length scale. One of the earliest use of continua with microstructure as a way to regularize the problem of strain localization was introduced by Ref. [22] who adopted a Cosserat type continuum, well suited for granular materials, to correctly model shear banding. This was soon followed with specific kinematically enriched models [23-25] and theoretical developments for classical and multiphase porous media and case studies for soils and concrete [26-32].

An alternative way is to use rate dependent models by adopting viscoplasticity and a time dependent viscosity parameter [33,34]. In this case, the band width depends not only on the material parameters but also on the load velocity.

A different approach is to treat the localization as a zero-thickness localization zone. A kinematic discontinuity of zero size is introduced via a displacement jump (strong discontinuity approach) leading to a singular deformation field, while the material behaviour is

\footnotetext{
* Corresponding author.

E-mail addresses: ibrahim.bitar@ec-nantes.fr (I. Bitar), panagiotis.kotronis@ec-nantes.fr (P. Kotronis), nathan.benkemoun@asn.fr (N. Benkemoun), stephane. grange@insa-lyon.fr (S. Grange).
} 
described via a cohesive law. This method belongs to the family of the Assumed Enhanced Strain Methods [35]. Strain localization occurs in the discontinuity and it is possible to define a dissipative mechanism which, in turn, allows to capture the same amount of dissipated energy regardless the size of the finite element. The approach complies therefore with the two efficiency criteria mentioned by Ref. [36]: ensuring objectivity with respect to the mesh size and taking into account the multi-scale nature of the problem under study. Several methods exist to effectively take into account such discontinuities. The main idea is to enhance the classical Finite Element Method (FEM) with additional degrees of freedom that carry the discontinuities and to make the mesh independent of their presence. A fine mesh or re-meshing is therefore not necessary to simulate crack propagation. The additional degrees of freedom can be carried either by the element nodes, Extended Finite Element Method (X-FEM), or integrated within the element, Embedded Finite Element Method (EFEM).

The X-FEM introduced in 1999 by Refs. [37-40], is based on the Partition of Unity Method (PUM) [41]. It was initially used with Griffith models and linear fracture mechanics and later for problems with discontinuity surfaces, cohesive models [42,43] and continuum mechanics constitutive laws. The discontinuity is interpolated with specific shape functions added to the classical finite element formulation. Cracking can pass through an element by cutting it in half, while the discontinuity variables are incorporated in the mesh at the cracked element nodes. The E-FEM, originally developed by Ref. [44] for modeling strain localization, is based on the Mixed Assumed Strain Approach and more particularly on the method of incompatible modes [35]. As in X-FEM, cracks can pass through the elements but the enhanced variables are in this case introduced at the element and not at the nodal level. This leads to two families of equilibrium equations, one at the global (structural) level and one at the local (element) level. This last one contains the enhanced variables. Static condensation is adopted and therefore the overall resolution at the structural scale remains the same as in the classical FEM. The E-FEM method can be therefore introduced straightforward to an existing finite element code without any change in its architecture.

[45] performed a comparative study on X-FEM and E-FEM. Both methods were implemented in the same finite element code to ensure a reliable comparison. The study shows that both methods provide similar results in terms of quality and quantity for a fairly fine mesh size. For coarse meshes however, E-FEM is generally more precise, while the convergence rate with increasing mesh refinement is slightly higher for X-FEM. From a computational point of view, modeling a single crack with X-FEM leads to a higher computational cost. For multiple cracks, the computational cost of E-FEM remains constant, while it linearly increases with the number of cracks for the X-FEM. The main disadvantage of the E-FEM is that the strain approximations in the two parts of the element separated by a discontinuity are not independent [46].

The objective of this article is to propose a novel generalized Timoshenko beam with higher order interpolation functions capable of reproducing the behaviour of structures up to failure. The Full-Cubic-Quadratic (FCQ) displacement-based Timoshenko beam proposed by Ref. [47] is adopted. This formulation uses shape functions of order three for the transverse displacements and two for the rotations and has an additional internal node. This results to a Timoshenko beam free of shear locking while one beam element provides the exact nodal displacements for whatever loading and suitable boundary conditions. Comparison of the FCQ beam with various Timoshenko beams found in the literature (see Refs. [48-50]) is studied in Ref. [51]. In Ref. [51], higher order interpolation functions are also adopted for the axial displacements in order to improve the axial force - moment coupling of the initial formulation [47] for non linear problems. The beam section is described through a stress- resultant constitutive model that takes the form of moment-curvature relation. In order to reproduce failure, a rotation discontinuity is adopted at the element level following the E-FEM method. For simplicity reasons and as a first step, only bending failure is thus considered in this paper; shear is elastic and shear failure problems are not addressed. The developments presented are also limited to geometrically linear Timoshenko beams. The equilibrium equations are formulated using the initial geometry of the structure and are not updated with the deformation. Validation is provided using classical civil engineering applications (i.e. failure or reinforced concrete structures).

The paper is organized as follows: in the second section, several already published generalized beam finite elements with embedded discontinuities are presented. In section three, the governing equations of the enhanced FCQ Timoshenko beam with embedded rotation discontinuity are detailed. The variational formulation is provided in the next section allowing the determination of the enhancement functions. In section five, the generalized constitutive models for the continuum and the cohesive parts are presented and in section six the computational procedures after linearization of the equilibrium equations and the implementation of the generalized laws. The paper ends with several numerical examples: a cantilever beam submitted to a rotation, to a transversal displacement and a two-storey reinforced concrete frame. These numerical examples illustrate the capacity of the novel beam element to regularize the results and to reproduce the structural behaviour up to failure.

\section{Enhanced generalized beams in the literature}

A generalized beam is a beam finite element with stress-resultant constitutive models [52-54]. In this category, we can also integrate the concept of macro-element introduced in geotechnics by Nova and Montrasio [55]. The term enhanced is used for beams with embedded discontinuities.

Armero and Ehrlich [14] were among the first to perform a failure analysis of steel structures using a generalized enhanced Timoshenko beam. The authors adopted elasto-plastic laws, introduced a strong discontinuity in the rotation field and adopted a cohesive dissipative law (moment-rotation jump) [56]. enhanced the three components of the displacement field (axial, transverse and rotation) and introduced three dissipative mechanisms (three cohesive models). Using an eigenvalue analysis, the authors have shown that high order enhancement functions are necessary to avoid shear locking [57]. studied Euler-Bernoulli beams and proposed two enhancements, the first for the axial displacement and the second for the rotation field. They showed that the choice of the discontinuity interpolating functions is crucial to obtain accurate results.

[58] combined an enhanced Euler-Bernoulli beam and a shell element to simulate failure of frame structures. The proposed EulerBernoulli beam differs from Ref. [57] as only the rotation component is enhanced [59]. and [60] proposed an enhanced Timoshenko beam for reinforced concrete structures. A multi-scale analysis, using a multifiber beam approach, was carried out to identify the parameters of the generalized model [61]. developed an Euler-Bernoulli beam with a bi-linear elasto-plastic behaviour for bending behaviour where only the rotation field was enhanced. Following $[59,62]$ presented a Timoshenko beam for reinforced concrete structures considering two rupture modes, shear and bending. The transverse and the rotational fields were therefore enhanced. Finally [63], presented a Timoshenko multifibre beam element with an axial displacement discontinuity. An elasticplastic behaviour was considered for steel and damage for concrete to reproduce the behaviour of reinforced concrete structures up to failure.

We present in the following how enhancement (embedded rotation discontinuity) can be taken into account in the novel FCQ Timoshenko beam element formulation [47]. 


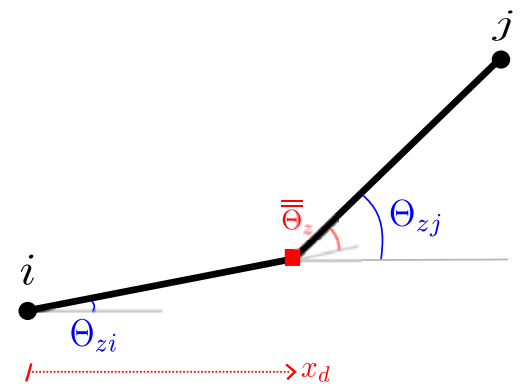

Fig. 1. Beam discontinuities kinematics, enhancement of the rotation field.

\section{Enhanced FCQ Timoshenko beam}

Consider a beam of length $€$ discretized with $n$ FCQ beam elements [47] $e=\left[x_{i} ; x_{j}\right]$ of length $L=x_{j}-x_{i}$ and external nodes $i$ and $j$. The generalized displacement vector $\mathbf{U}_{S}(x, t)$ is approximated by an equation of the form $\mathbf{U}_{S}(x, t)=\mathbf{N}(x) \mathbf{d}_{e}(t)$, where $\mathbf{d}_{e}(t)$ is a vector containing the external nodal displacements of the element $e$ and $\mathbf{N}$ is the matrix of the shape functions depending only on $x$, the beam axis. For sake of simplicity, presentation is made hereafter in 2D.

$\mathbf{U}_{S}(x, t)=\left[\begin{array}{c}U_{x}(x, t) \\ U_{y}(x, t) \\ \Theta_{z}(x, t)\end{array}\right]=\left[\begin{array}{l}\mathbf{N}^{u}(x) \mathbf{d}_{e}(t) \\ \mathbf{N}^{v}(x) \mathbf{d}_{e}(t) \\ \mathbf{N}^{\theta}(x) \mathbf{d}_{e}(t)\end{array}\right]$

with $\mathbf{N}^{u}, \mathbf{N}^{v}$ and $\mathbf{N}^{\theta}$ the shape functions of the three components defined by Ref. [47]:

$\left[\begin{array}{l}\mathbf{N}^{u}(x) \\ \mathbf{N}^{v}(x) \\ \mathbf{N}^{\theta}(x)\end{array}\right]=\left[\begin{array}{ccccccccc}N_{1}^{u} & 0 & 0 & 0 & 0 & 0 & N_{2}^{u} & 0 & 0 \\ 0 & N_{2}^{v} & 0 & N_{7}^{v} & 0 & N_{9}^{v} & 0 & N_{5}^{v} & 0 \\ 0 & 0 & N_{3}^{\theta} & 0 & N_{8}^{\theta} & 0 & 0 & 0 & N_{6}^{\theta}\end{array}\right]$,

where

$$
\begin{aligned}
& N_{1}^{u}=1-\frac{x}{L} \quad N_{7}^{v}=2\left(1-\frac{x}{L}\right)^{2}\left(\frac{x}{L}\right) \quad N_{3}^{\theta}=\left(1-\frac{x}{L}\right)\left(1-3 \frac{x}{L}\right) \\
& N_{2}^{u}=\frac{x}{L} \quad N_{9}^{v}=-2\left(\frac{x}{L}\right)^{2}\left(1-\frac{x}{L}\right) \quad N_{8}^{\theta}=1-\left(1-2 \frac{x}{L}\right)^{2} \\
& N_{2}^{v}=\left(1-\frac{x}{L}\right)^{2}\left(1+2 \frac{x}{L}\right) \quad N_{5}^{v}=\left(\frac{x}{L}\right)^{2}\left(3-2 \frac{x}{L}\right) \quad N_{6}^{\theta}=-\left(\frac{x}{L}\right)\left(2-3 \frac{x}{L}\right)
\end{aligned}
$$

$\mathbf{d}_{e}$ is the nodal displacement vector defined by:

$$
\mathbf{d}_{e}=\left[\begin{array}{lllllllll}
U_{x i} & V_{y i} & \Theta_{z i} & \Delta V_{y k}^{1} & \Delta \Theta_{z k} & \Delta V_{y k}^{2} & U_{x j} & V_{y j} & \Theta_{z j}
\end{array}\right]^{T}
$$

where $\Delta V_{y k}^{1}, \Delta \Theta_{z k}$ and $\Delta V_{y k}^{2}$ are the degrees of freedom of the internal node (with no specific physical meaning) [47]. It should be mentioned that only the enhancement of the rotation field is considered hereafter, we are dealing primarily with bending problems, see Fig. 1. This is why first degree polynomial shape functions $\left(N_{1}^{u}, N_{2}^{u}\right)$ are considered for the axial displacements (3). As already mentioned however, higher order interpolation functions for the axial displacements can be adopted for the FCQ Timoshenko beam in order to improve the axial force - moment coupling, for more details see Ref. [51]. This does not change the developments presented in this paper.

The enhanced rotation field is written as follows:

$\Theta_{z}(x, t)=\mathbf{N}^{\theta}(x) \mathbf{d}_{e}(t)+M(x) \overline{\bar{\Theta}}_{e}(t)$

where $\overline{\bar{\Theta}}_{e}(t)$ represents the discontinuity variable of the rotation field and $M(x)$ the enhancement function defined as follows:

$M(x)=\bar{M}(x)+H_{x_{d}}$

with $x_{d}$ the position of the discontinuity within the element and $H_{x_{d}}$ the Heaviside function defined as:

$H_{x_{d}}(x)=\left\{\begin{array}{lll}1 & \text { for } & x>x_{d} \\ 0 & \text { for } & x<x_{d}\end{array}\right.$

It remains therefore to define the function $\bar{M}(x)$ and the position of the discontinuity within the element. This requires a good understanding of the beam discontinuity kinematics [57]. Indeed, total failure is reached when there is no more stress transfer through the discontinuity, in our case if the value of the moment at discontinuity is zero [57]. refer to this final deformation state as zero hinge mode.

\subsection{Modification of the rotation field shape functions}

The enhanced rotation field should reproduce correctly the rotations at the three nodes of the FCQ element (the two external nodes $i, j$ and the internal node $k$ ). In the original FCQ Timoshenko beam formulation however, the internal node has not a specific physical meaning, $\Delta \Theta_{z k}(t)$ does not represent the rotation in the middle $\left(x=\frac{L}{2}\right)$ of the element [47]. We propose hereafter a slight modification of the interpolation of the rotation field that does not alter the performance of the FCQ beam, it is just used in order to correctly identify the function $\bar{M}(x)$. The main idea is to provide a physical sense to the internal rotation degree of freedom, the rotation in the middle of the element $\left(x=\frac{L}{2}\right)$, see Fig. 2.

The rotation in the middle of the element $\Theta_{z}\left(\frac{L}{2}, t\right)$ can be expressed as a function of the original FCQ shape functions $N_{3}^{\theta}, N_{6}^{\theta}$ and $N_{8}^{\theta}$ as follows:

$\Theta_{z}\left(\frac{L}{2}, t\right)=\Theta_{z k}(t)=\Delta \Theta_{z k}(t)-\frac{1}{4}\left[\Theta_{z i}(t)+\Theta_{z j}(t)\right]$

with $k$ the index of the internal node positioned in the middle of the FCQ element. The rotation field can be therefore interpolated in the following way:

$$
\begin{aligned}
\Theta_{z}(x, t) & =N_{3}^{\theta}(x) \Theta_{z i}+N_{6}^{\theta}(x) \Theta_{z j}+N_{8}^{\theta}(x) \Delta \Theta_{z k} \\
& =N_{3}^{\theta}(x) \Theta_{z i}+N_{6}^{\theta}(x) \Theta_{z j}+N_{8}^{\theta}(x)\left(\Theta_{z k}+\frac{1}{4}\left[\Theta_{z i}(t)+\Theta_{z j}(t)\right]\right) \\
& =\left(N_{3}^{\theta}(x)+\frac{1}{4} N_{8}^{\theta}(x)\right) \Theta_{z i}+\left(N_{6}^{\theta}(x)+\frac{1}{4} N_{8}^{\theta}(x)\right) \Theta_{z j}+N_{8}^{\theta}(x) \Theta_{z k} \\
& =N_{3^{*}}^{\theta}(x) \Theta_{z i}+N_{6}^{\theta}(x) \Theta_{z j}+N_{8^{*}}^{\theta}(x) \Theta_{z k}
\end{aligned}
$$

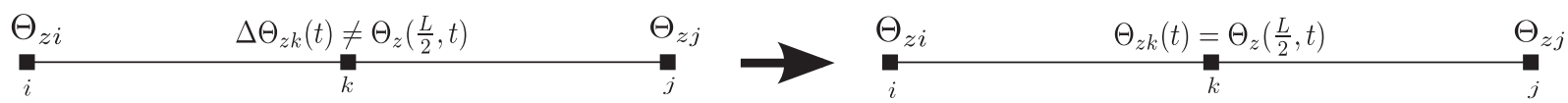

Fig. 2. Modified FCQ element. 


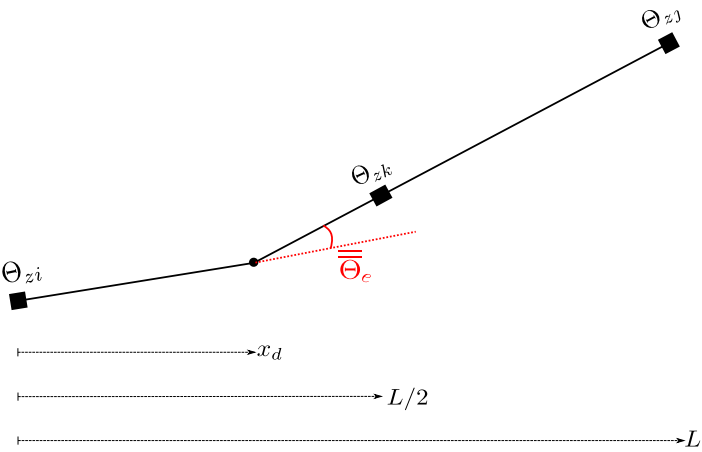

(a) Case $x_{d}<\frac{L}{2}$ with $\Theta_{z k}=\Theta_{z j}$

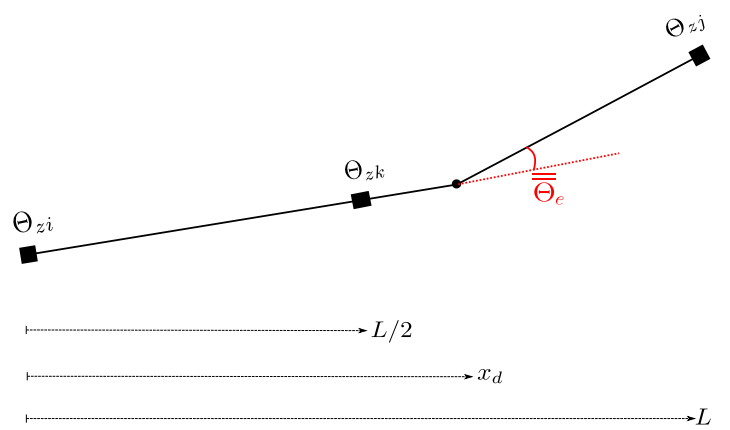

(b) Case $x_{d}>\frac{L}{2}$ with $\Theta_{z k}=\Theta_{z i}$

Fig. 3. Zero hinge mode (fully opened discontinuity).

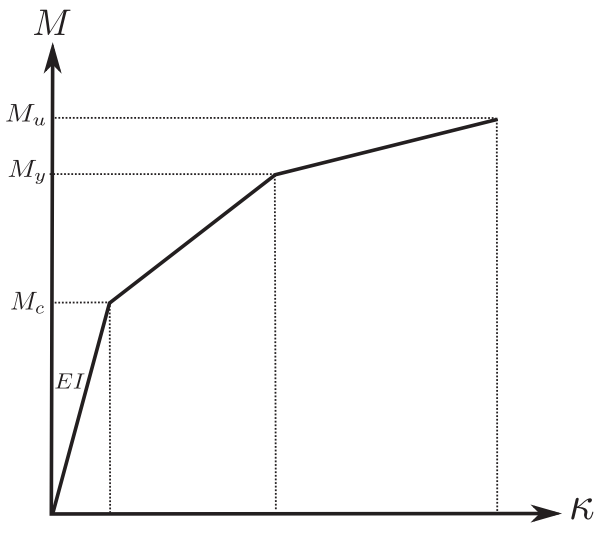

(a) Moment curvature continuous model

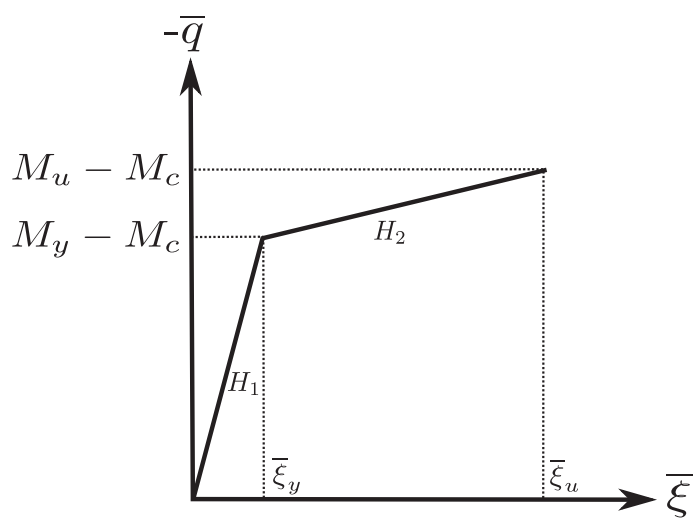

(b) Internal hardening variable

Fig. 4. Continuous model.

with the three new interpolation functions:

$$
\begin{aligned}
& N_{3^{*}}^{\theta}(x)=N_{3}^{\theta}(x)+\frac{1}{4} N_{8}^{\theta}(x)=1-\frac{3 x}{L}+\frac{2 x^{2}}{L^{2}} \\
& N_{6^{*}}^{\theta}(x)=N_{6}^{\theta}(x)+\frac{1}{4} N_{8}^{\theta}(x)=-\frac{x}{L}+2 \frac{x^{2}}{L^{2}} \\
& N_{8^{*}}^{\theta}(x)=N_{8}^{\theta}(x)=\frac{4 x}{L}-\frac{4 x^{2}}{L^{2}}
\end{aligned}
$$

These new shape functions (10) are associated with the three nodal rotation values located at $x=0, x=L$ and $x=\frac{L}{2}$ and can now be used to correctly identify the function $\bar{M}(x)$. They verify the classical conditions that must be respected by the shape functions [64]:

$$
\begin{aligned}
& N_{3^{*}}^{\theta}(0)=1 \quad, \quad N_{3^{*}}^{\theta}\left(\frac{L}{2}\right)=0 \quad, \quad N_{3^{*}}^{\theta}(L)=0 \\
& N_{6^{*}}^{\theta}(0)=0 \quad, \quad N_{6^{*}}^{\theta}\left(\frac{L}{2}\right)=0 \quad, \quad N_{6^{*}}^{\theta}(L)=1 \\
& N_{8^{*}}^{\theta}(0)=0 \quad, \quad N_{8^{*}}^{\theta}\left(\frac{L}{2}\right)=1 \quad, \quad N_{8^{*}}^{\theta}(L)=0 \\
& \text { and } \sum_{\alpha=3,6,8} N_{\alpha^{*}}^{\theta}(x)=1
\end{aligned}
$$

The expression of the enhanced rotation field is therefore finally written as:

$\Theta_{z}(x, t)=N_{3^{*}}^{\theta}(x) \Theta_{z i}+N_{6^{*}}^{\theta}(x) \Theta_{z j}+N_{8^{*}}^{\theta}(x) \Theta_{z k}+\left[\bar{M}(x)+H_{x_{d}}\right] \overline{\bar{\Theta}}_{e}(t)$

\subsection{Identification of the function $\bar{M}(x)$}

The presence of a discontinuity within a beam element must not affect the compatibility between different elements. In other words, the nodal rotation values at points $i$ and $j$ must not be affected. Following (12), the following conditions must be met:

$$
\begin{array}{ll}
\text { For } x=0 & : \quad \Theta_{z}(0, t)=\Theta_{z i} \Longrightarrow \bar{M}(0)=0 \\
\text { For } x=L & : \quad \Theta_{z}(L, t)=\Theta_{z j} \Longrightarrow \bar{M}(L)=-1
\end{array}
$$

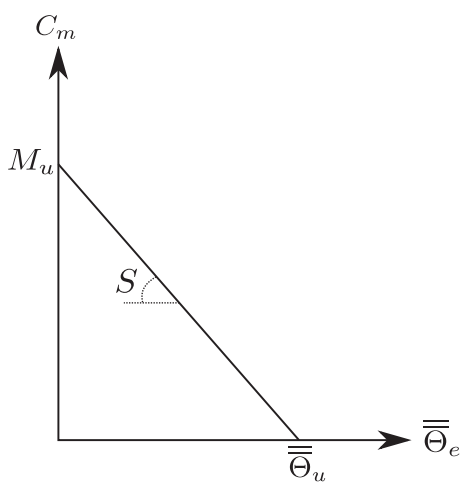

Fig. 5. Cohesive model. 


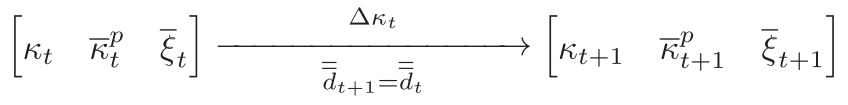

Fig. 6. Path I: Initial and final states.

$$
\begin{gathered}
{\left[\begin{array}{ll}
\kappa_{t} & \overline{\bar{\Theta}}_{e, t}
\end{array}\right] \stackrel{\Delta \kappa_{t}}{\bar{\kappa}_{t+1}^{p}}=\bar{\kappa}_{t}^{p}} \\
\bar{\xi}_{t+1}=\bar{\xi}_{t}
\end{gathered}
$$

Fig. 7. Path II: Initial and final states.

The modified FCQ Timoshenko beam has a third internal degree of freedom carried by a node located in the middle of the element $\left(x=\frac{L}{2}\right)$, see Fig. 2. Two cases are studied following the position of the discontinuity $x_{d}\left(x_{d} \leq \frac{L}{2}\right.$ or $\left.x_{d}>\frac{L}{2}\right)$ :

$\Theta_{z}\left(\frac{L}{2}, t\right)=\Theta_{z k} \Longrightarrow\left\{\begin{array}{l}\text { si } x_{d} \leq \frac{L}{2} \Longrightarrow \bar{M}\left(\frac{L}{2}\right)=-1 \\ \text { si } x_{d}>\frac{L}{2} \Longrightarrow \bar{M}\left(\frac{L}{2}\right)=0\end{array}\right.$

Several functions satisfy the conditions of equations (13) and (14). Nevertheless, in order to ensure order compatibility between the enhancement functions $\bar{M}(x)$ and the rotation interpolation functions $\left(N_{3^{*}}^{\theta}(x)\right.$, $N_{6^{*}}^{\theta}(x)$ and $\left.N_{8^{*}}^{\theta}(x)\right), \bar{M}(x)$ have to be quadratic. If this condition is not met, numerical difficulties may appear [51,65]. The quadratic functions that satisfy the conditions (13) and (14) are:

$\bar{M}(x)=\left\{\begin{array}{lr}-\left(N_{6^{*}}^{\theta}(x)+N_{8^{*}}^{\theta}(x)\right)=-\frac{3 x}{L}+\frac{2 x^{2}}{L^{2}} & \text { for } \quad x_{d} \leq \frac{L}{2} \\ -N_{6^{*}}^{\theta}(x)=\frac{x}{L}-\frac{2 x^{2}}{L^{2}} & \text { for } \quad x_{d}>\frac{L}{2}\end{array}\right.$

\subsection{Derivation of the function $\bar{G}_{r}(x)$ and discontinuity position}

Deformation can be obtained by derivation as follows:

$\varepsilon_{S}(x, t)=\left\{\begin{array}{l}\varepsilon(x, t)=\frac{\partial}{\partial x} U_{x}(x, t) \\ \gamma(x, t)=\frac{\partial}{\partial x} V_{y}(x, t)-\Theta_{z}(x, t), \\ \kappa(x, t)=\frac{\partial}{\partial x} \Theta_{z}(x, t)\end{array}\right.$

with $\varepsilon_{S}(x, t)$ the vector of the generalized deformations of the section, $\varepsilon(x, t), \gamma(x, t)$ and $\kappa(x, t)$ respectively the axial deformation, shear defor-

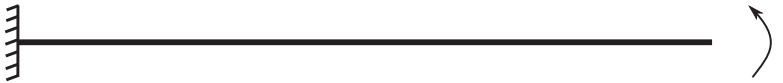

$\Delta \Theta_{z}$

Fig. 8. Cantilever beam structure subjected to a rotation.

mation and curvature. Therefore,

$\varepsilon_{S}(x, t)=\left\{\begin{array}{l}\varepsilon(x, t)=\mathbf{B}^{\varepsilon}(x) \mathbf{d}_{e}(t) \\ \gamma(x, t)=\mathbf{B}^{\gamma}(x) \mathbf{d}_{e}(t) \\ \kappa(x, t)=\mathbf{B}^{\kappa}(x) \mathbf{d}_{e}(t)+G_{r}(x) \overline{\bar{\Theta}}_{e}(t)\end{array}\right.$,

with $\mathbf{B}^{\varepsilon}(x), \mathbf{B}^{\gamma}(x)$ and $\mathbf{B}^{K}(x)$ the derivatives of the shape functions for the three deformation components and $G_{r}(x)$ the derivative of the enhancement function $M(x)$

$G_{r}(x)=\bar{G}_{r}(x)+\delta_{x_{d}}$,

with $\delta_{x_{d}}$ the Dirac delta function at $x_{d}\left(\delta_{x_{d}}=\infty\right.$ for $x=x_{d}$ and $\delta_{x_{d}}=0$ otherwise) and $\bar{G}_{r}(x)=\frac{\partial \bar{M}(x)}{\partial x}$ calculated as follows:

$\bar{G}_{r}(x)= \begin{cases}-\left(B_{6^{*}}^{K}(x)+B_{8^{*}}^{K}(x)\right)=\frac{4 x}{L^{2}}-\frac{3}{L} & \text { for } \quad x_{d} \leq \frac{L}{2} \\ -B_{6^{*}}^{\kappa}(x)=-\frac{4 x}{L^{2}}+\frac{1}{L} & \text { for } \quad x_{d}>\frac{L}{2}\end{cases}$

Hence, the enhanced curvature is divided into a regular $(\widetilde{\kappa}(x, t))$ and a singular $(\overline{\bar{\kappa}}(x, t))$ :

$\kappa(x, t)=\underbrace{\mathbf{B}^{\kappa}(x) \mathbf{d}_{e}(t)+\bar{G}_{r}(x) \overline{\bar{\Theta}}_{e}(t)}_{\widetilde{\kappa}(x, t)}+\underbrace{\delta_{x_{d}} \overline{\bar{\Theta}}_{e}(t)}_{\overline{\bar{\kappa}}(x, t)}$

The function $\bar{M}(x)$ has been previously determined from the three rotation nodal values of the FCQ element. The $\bar{G}_{r}(x)$ function should be checked if it is compatible with the zero hinge mode $(\widetilde{\kappa}(x, t)=0)$, corresponding to a fully opened discontinuity. The following kinematic relations correspond to the zero hinge mode, Fig. 3:

for $x_{d} \leq \frac{L}{2} \quad$ (figure 3a) : $\Theta_{z k}=\Theta_{z j}$ and $\overline{\bar{\Theta}}_{e}=\Theta_{z k}-\Theta_{z i}$

for $x_{d}>\frac{L}{2} \quad$ (figure 3b) : $\Theta_{z k}=\Theta_{z i}$ and $\overline{\bar{\Theta}}_{e}=\Theta_{z j}-\Theta_{z k}$

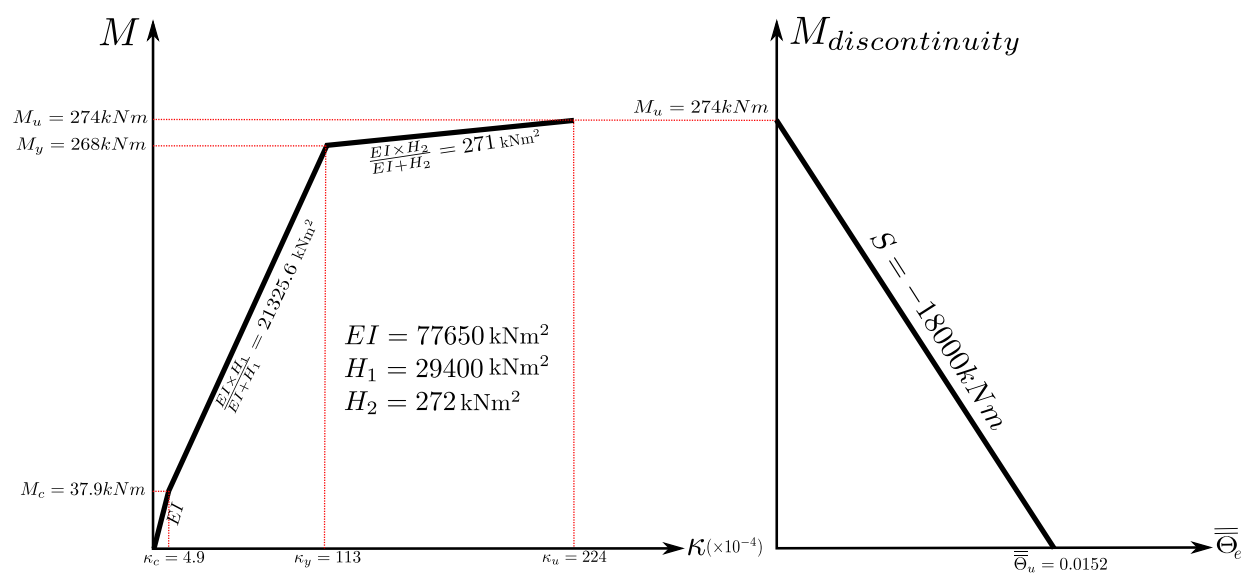

Fig. 9. Cantilever beam structure subjected to a rotation - Elasto-plastic model with bilinear hardening and embedded discontinuity behaviour. 
For $x_{d} \leq \frac{L}{2}$, using equations (19) and (21) the regular part of the curvature can be expressed as:

$$
\begin{aligned}
\tilde{\kappa}(x, t)= & \mathbf{B}^{\kappa}(x) \mathbf{d}_{e}(t)+\bar{G}_{r}(x) \overline{\bar{\Theta}}_{e}(t) \\
= & B_{3^{*}}^{\kappa}(x) \Theta_{z i}(t)+B_{6^{*}}^{\kappa}(x) \Theta_{z j}(t)+B_{8^{*}}^{\kappa}(x) \Theta_{z k}(t) \\
& -\left[B_{6^{*}}^{\kappa}(x)+B_{8^{*}}^{\kappa}(x)\right]\left(\Theta_{z k}(t)-\Theta_{z i}(t)\right)
\end{aligned}
$$

As $\Theta_{z k}=\Theta_{z j}$ (Fig. 3a) finally we get:

$$
\widetilde{\kappa}(x, t)=\underbrace{\left(B_{3^{*}}^{\kappa}(x)+B_{6^{*}}^{\kappa}(x)+B_{8^{*}}^{\kappa}(x)\right)}_{=0 \text { see }(11)} \Theta_{z i}(t)=0,
$$

Equation (23) is always verified as $B_{3^{*}}^{\kappa}(x)+B_{6^{*}}^{\kappa}(x)+B_{8^{*}}^{\kappa}(x)=0$, due to the properties of the shape functions, see (11). If now we repeat the same procedure for the case where $x_{d}>\frac{L}{2}$, this time using $\Theta_{z k}=\Theta_{z i}$ (Fig. 3b), it can be again found that $\widetilde{\kappa}(x, t)=0$. Equation (19) verifies therefore the zero hinge mode state. The discontinuity position can be determined considering the force distribution within the FCQ element. A discontinuity is generated at the point $x_{d}$ where the force (bending moment) reaches its ultimate value. Since the rotation interpolation functions of the FCQ element $\mathbf{N}^{\theta}$ are quadratic, the curvature is linear and therefore the maximum curvature appears at the ends of the element at $x=0$ or at $x=L$. For the specific case where the curvature is constant (pure bending), deformation must be constant and therefore the linear forms of the enhancement function $\bar{G}_{r}(x)$ (19) are no longer appropriate. For this specific case, a constant function is therefore assumed, $\bar{G}_{r}(x)=-\frac{1}{L}$. The discontinuity can appear at any position within the element, we decide arbitrarily to fix it in the middle at $x_{d}=\frac{L}{2}$.

The function $\bar{G}_{r}(x)$ finally takes the following form depending on the position $x_{d}$ of the discontinuity:

$\bar{G}_{r}(x)=\left\{\begin{array}{lll}\frac{4 x}{L^{2}}-\frac{3}{L} & \text { for } & x_{d}=0 \\ -\frac{4 x}{L^{2}}+\frac{1}{L} & \text { for } & x_{d}=L \\ -\frac{1}{L} & \text { for } & x_{d}=\frac{L}{2}\end{array}\right.$

\section{Variational formulation}

The Principle of Virtual Work for a beam structure discretized with $n$ FCQ beam elements $e$ of length $L$ is written as:

$\sum_{e}\left\{W_{\text {int }}^{e}-W_{\text {ext }}^{e}\right\}=0$

with $W_{\text {int }}^{e}$ and $W_{e x t}^{e}$ the work of the internal and external forces on the element $e$ respectively.

The work of the internal forces on the element $e$ becomes:

$W_{\text {int }}^{e}=\int_{L} \delta \varepsilon_{S}^{*}(x, t)^{T} \mathbf{F}_{S}(x, t) d x$

with $\delta$ the variation symbol, $(\bullet)^{*}$ the symbol that indicates that $(\bullet)$ takes a virtual value, $\varepsilon_{S}^{*}(x, t)$ the vector of the virtual generalized deformations and $\mathbf{F}_{S}(x, t)$ the vector of the generalized forces of the section defined as follows:

$\varepsilon_{S}^{*}(x, t)=\left[\begin{array}{l}\varepsilon^{*}(x, t) \\ \gamma^{*}(x, t) \\ \kappa^{*}(x, t)\end{array}\right]$ and $\mathbf{F}_{S}(x, t)=\left[\begin{array}{c}F_{x}(x, t) \\ F_{y}(x, t) \\ M_{z}(x, t)\end{array}\right]$

$F_{x}(x, t), F_{y}(x, t)$ and $M_{z}(x, t)$ are respectively the axial force, the shear force and the bending moment of the section.

The virtual displacements and virtual deformations are interpolated with the same shape functions as the real ones. An enhanced shape function $G_{v}(x)$ is used for the virtual discontinuity variables (see section 4.1 for its identification). $G_{v}(x)$ plays the same role as the $G_{r}(x)$ function of section 3.3 associated with the real discontinuity variables. It should be noted however that the enhanced shape functions $G_{r}$ and $G_{v}$ are generally different. The real discontinuity is interpolated following kinematic considerations (section 3.2) while the virtual discontinuity is interpolated following static considerations (section 4.1). Both interpolations in the same formulation are first proposed in Refs. [44] and [66].

The virtual enhanced curvature variation $\kappa^{*}(x, t)$ is (see also equation (18)):

$$
\begin{aligned}
\delta \kappa^{*}(x, t) & =\mathbf{B}^{\kappa}(x) \delta \mathbf{d}_{e}^{*}(t)+G_{\nu}(x) \delta \overline{\bar{\Theta}}_{e}^{*}(t) \\
& =\mathbf{B}^{\kappa}(x) \delta \mathbf{d}_{e}^{*}(t)+\bar{G}_{\nu}(x) \delta \overline{\bar{\Theta}}_{e}^{*}(t)+\delta_{x_{d}} \delta \overline{\bar{\Theta}}_{e}^{*}(t)
\end{aligned}
$$

with $\delta \overline{\bar{\Theta}}_{e}^{*}(t)$ the variation of the virtual rotational discontinuity of the element $e$. Introducing equation (28) in (26) gives:

$W_{\text {int }}^{e}=\delta \mathbf{d}_{e}^{*}(t)^{T} \mathbf{F}_{\mathrm{int}, B}^{e}(t)+\delta \overline{\bar{\Theta}}_{e}^{*}(t) F_{\mathrm{int}, G}^{e}(t)$

with $\mathbf{F}_{\mathrm{int}, B}^{e}(t)$ and $F_{\mathrm{int}, G}^{e}(t)$ the elementary nodal internal forces defined as:

$\mathbf{F}_{\text {int }, B}^{e}(t)=\int_{L_{L}} \mathbf{B}^{T}(x) \mathbf{F}_{S}(x, t) d x$

$F_{\mathrm{int}, G}^{e}(t)=\int_{L} G_{v}(x) M_{z}(x, t) d x$

The work of the external forces on the element $e$ becomes:

$W_{e x t}^{e}=\delta \mathbf{d}_{e}^{*}(t)^{T} \mathbf{F}_{e x t}^{e}(t)$

with $\mathbf{F}_{\text {ext }}^{e}(t)$ the vector of the external forces at the element level.

Introducing equations (29) and (31) in the virtual works principle (25) makes possible to obtain the balance equation of the structure in the following form:

$$
\begin{aligned}
& \sum_{e}\left\{\delta \mathbf{d}_{e}^{*}(t)^{T} \mathbf{F}_{\mathrm{int}, B}^{e}(t)+\delta \overline{\bar{\Theta}}_{e}^{*}(t) F_{\mathrm{int}, G}^{e}(t)-\delta \mathbf{d}_{e}^{*}(t)^{T} \mathbf{F}_{e x t}^{e}(t)\right\}=0 \\
\Leftrightarrow & \delta \mathbf{d}_{s t r}^{*}(t)^{T}\left(\mathbf{F}_{\mathrm{int}, B}^{\mathrm{str}}(t)-\mathbf{F}_{\text {ext }}^{\mathrm{str}}(t)\right)+\sum_{e}^{\overline{\bar{n}}} \delta \overline{\bar{\Theta}}_{e}^{*}(t) F_{\mathrm{int}, G}^{e}(t)=0
\end{aligned}
$$

with $\mathbf{d}_{s t r}^{*}$ the global displacement vector assembling all the virtual nodal degrees of freedom and $\overline{\bar{n}}(\leq n)$ the number of elements where the rotation discontinuity is active.

Furthermore, the equilibrium must be respected for all virtual displacements $\delta \mathbf{d}_{s t r}(t)$ as well as for any elementary virtual jump $\overline{\bar{\Theta}}_{e}(t)$. Therefore, we get the following system:

$\mathbf{F}_{\text {int }, B}^{s t r}(t)-\mathbf{F}_{\text {ext }}^{s t r}(t)=0$

$\forall e \in 1,2, \ldots, \overline{\bar{n}}: F_{\text {int }, G}^{e}(t)=0$

The first equation in the system (33) represents the overall equilibrium of the structure, with $\mathbf{F}_{\text {int }, B}^{\text {str }}(t)$ and $\mathbf{F}_{\text {ext }}^{\text {str }}(t)$ the internal and external forces at the structural level respectively. The size of these vectors is equal to the number of degrees of freedom per node ( 3 for a 2D problem) multiplied by the total number of nodes. The second equation in (33) represents the local equilibrium at the level of each rotational discontinuity inside the element. Considering the decomposition of $G_{\nu}(x)$ in two parts $\bar{G}_{v}(x)$ and $\overline{\bar{G}}_{v}(x)=\delta_{x_{d}}, F_{\text {int }, G}^{e}(t)$ becomes:

$$
\begin{aligned}
F_{\text {int }, G}^{e}(t) & =\int_{L} G_{v}(x) M_{z}(x, t) d x=\int_{L}\left(\bar{G}_{v}(x)+\overline{\bar{G}}_{v}(x)\right) M_{z}(x, t) d x \\
& =\int_{L} \bar{G}_{v}(x) M_{z}(x, t) d x+\int_{L} \overline{\bar{G}}_{v}(x) M_{z}(x, t) d x=0
\end{aligned}
$$


and as a result

$\int_{L} \bar{G}_{v}(x) M_{z}(x, t) d x=-\int_{L} \delta_{x_{d}} M_{z}(x, t) d x=-M_{z}\left(x_{d}, t\right)=-C_{m}(t)$

where $C_{m}(t)$ is the cohesive bending moment at $x_{d}$.

\subsection{Identification of the function $\bar{G}_{v}$}

As already mentioned, the enhanced shape function $\bar{G}_{v}(x)$ is used for the virtual discontinuity variables and plays the same role as the function $\bar{G}_{r}(x)$ associated with the real discontinuity variables. The function $\bar{G}_{v}(x)$ is often considered equal to $\bar{G}_{r}(x)$. This however is not possible for the enhanced FCQ Timoshenko beam element formulation as shown hereafter.

At the discontinuity position $x_{d}$, the equality (34) between the cohesive bending moment $C_{m}(t)$ and the generalized bending moment $M_{z}\left(x_{d}, t\right)$ should be verified. Since the moment takes a linear form (see section 3.3), it can be expressed as:

$M_{z}(x, t)=\alpha x+\beta$

with $\alpha$ and $\beta$ constants. For the case where $x_{d}=0$, we have $\bar{G}_{r}(x)=$ $\frac{4 x}{L^{2}}-\frac{3}{L}$ (24). Considering $\bar{G}_{v}(x)=\bar{G}_{r}(x)$ and introducing the latter in (34) we get:

$$
\begin{aligned}
C_{m}(t) & =-\int_{L} \underbrace{\bar{G}_{v}(x)}_{=\bar{G}_{r}(x)} M_{z}(x, t) d x \\
& =-\int_{L}\left(\frac{4 x}{L^{2}}-\frac{3}{L}\right)(\alpha x+\beta) d x=\frac{5 L}{3} \alpha-2 \beta \neq \beta=M_{z}(0, t)
\end{aligned}
$$

In other words and for $x_{d}=0$, the equilibrium condition (34) is not verified if $\bar{G}_{v}(x)$ is considered equal to $\bar{G}_{r}(x)$. The way to correctly identify $\bar{G}_{v}(x)$ is to consider the local equilibrium condition (34) and the Patch test.

\subsubsection{Local equilibrium condition}

Let's assume the following linear form:

$\bar{G}_{v}(x)=c x+d$

with $c$ and $d$ constants to be determined by verifying the local equilibrium condition $C_{m}(t)=M_{z}\left(x_{d}, t\right)$. We get:

$$
\begin{aligned}
C_{m}(t) & =-\int_{L} \bar{G}_{v}(x) M_{z}(x, t) d x=-\int_{L}(c x+d)(\alpha x+\beta) d x \\
& =\alpha\left(-c \frac{L^{3}}{3}-d \frac{L^{2}}{2}\right)+\beta\left(-c \frac{L^{2}}{2}-d L\right)
\end{aligned}
$$

and

$M_{z}\left(x_{d}, t\right)=\alpha x_{d}+\beta$

The equality of the equations (38) and (39) gives the following results:

$\alpha x_{d}+\beta=\alpha\left(-c \frac{L^{3}}{3}-d \frac{L^{2}}{2}\right)+\beta\left(-c \frac{L^{2}}{2}-d L\right)$

The identification of the two sides of the equation (40) provides:

$c=\frac{6}{L^{2}}-\frac{12}{L^{3}} x_{d}$

$d=-\frac{4}{L}+\frac{6}{L^{2}} x_{d}$

and the enhancement function $\bar{G}_{v}(x)$ associated with a discontinuity at $x_{d}$ becomes:

$\bar{G}_{v}(x)=\left(\frac{6}{L^{2}}-\frac{12}{L^{3}} x_{d}\right) x-\frac{4}{L}+\frac{6}{L^{2}} x_{d}$
Finally, the enhancement function $\bar{G}_{v}(x)$ of the virtual rotational discontinuity takes the following forms depending on the position $x_{d}$ of the discontinuity inside the FCQ Timoshenko beam element:

$\bar{G}_{v}(x)= \begin{cases}\frac{6 x}{L^{2}}-\frac{4}{L} & \text { for } \quad x_{d}=0 \\ -\frac{6 x}{L^{2}}+\frac{2}{L} & \text { for } \quad x_{d}=L \\ -\frac{1}{L} & \text { for } \quad x_{d}=\frac{L}{2}\end{cases}$

\subsubsection{Patch test}

An additional verification is required to finally validate the $\bar{G}_{v}(x)$ function. The enhancement with integrated discontinuities is similar to the incompatible modes method and therefore the enhancement function should verify the Patch test [67]. This test, initially proposed by Ref. [68], reflects a convergence condition when refining the mesh. A large number of elements results in small dimension elements and consequently elementary strains and stresses should converge to constant values. The Patch test guarantees the ability to represent a constant state of stress per element [69].

The application of the Patch test requires that the additional virtual work associated with the enhancement is zero if the bending moment is constant along the element:

$$
\begin{array}{r}
\delta \overline{\bar{\Theta}}_{e}(t) F_{\mathrm{int}, G}^{e}(t)=0 \\
\delta \overline{\bar{\Theta}}_{e}(t) \int_{L} G_{v}(x) M_{z}(x, t) d x=0 \\
\text { Since } M_{z} \text { is constant per element } \Rightarrow \underbrace{\delta \overline{\bar{\Theta}}_{e}(t) M_{z}(x, t)}_{\neq 0} \int_{L} G_{v}(x) d x=0 \\
\Rightarrow \int_{L} G_{v}(x) d x=0
\end{array}
$$

The introduction of the two components (regular and singular) of $G_{v}(x)$ gives:

$\int_{L}\left(\bar{G}_{v}(x)+\overline{\bar{G}}_{v}(x)\right) d x=\int_{L}\left(\bar{G}_{v}(x)+\delta_{x_{d}}\right) d x=0$

Therefore and in order to verify the Patch test the condition is:

$\int_{L} \bar{G}_{v}(x) d x=-1$

Using the expressions of $\bar{G}_{v}(x)$ provided in (43) it can be verified that the Patch test (46) is satisfied.

\section{Generalized models}

A simple stress resultant model is considered hereafter, where the generalized forces (axial force, shear force and bending moment) are expressed respectively as a function of the generalized deformations (axial deformation, shear deformation and curvature). The axial and transverse behaviour are supposed elastic:

$F_{x}(x, t)=E A \varepsilon(x, t) \quad F_{y}(x, t)=k G A \gamma(x, t)$

where $E$ the Young modulus, $G$ the shear modulus, $A$ the section area and $k$ the shear correction factor [70].

For the bending behaviour, a continuous/cohesive coupled model formulated within the framework of the generalized standard materials [71] is introduced. The variables associated with the continuous model are surmounted by a bar, while the variables of the cohesive model by a double bar. Variables with no bars are associated with the coupling of the two models. 


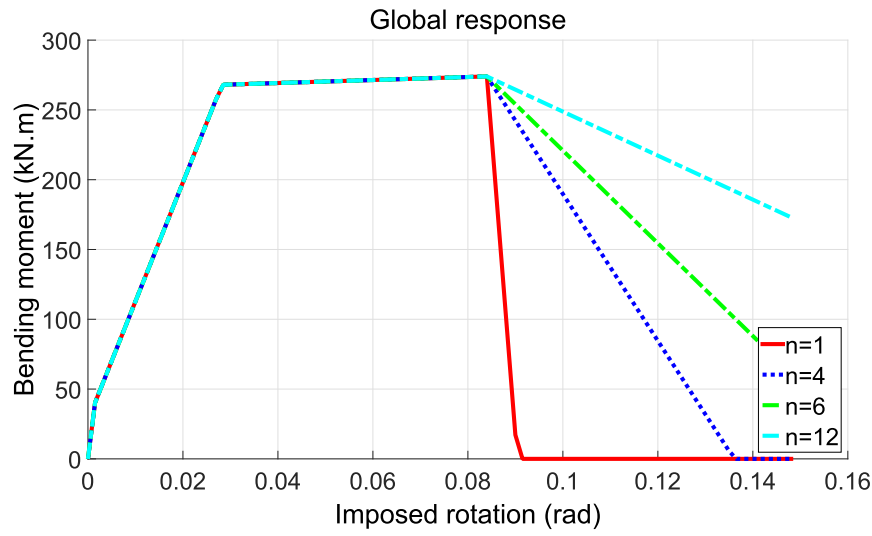

Fig. 10. Cantilever beam structure subjected to a rotation - Global response (bending moment - imposed rotation), FCQ elements with the same material characteristics.

\subsection{Continuous model}

A generalized elasto-plastic model with an isotropic bi-linear hardening (Fig. 4) is adopted. This simple model is chosen as it is sufficient for several civil engineering applications (see section 7). Actually, it allows reproducing the three main phases of the reinforced concrete behaviour (elastic, concrete damage, steel plastification). The development of the generalized law follows the classical framework of plasticity. More specifically:

- The generalized continuous curvature $\bar{\kappa}(x, t)$ is partitioned into an elastic $\bar{\kappa}^{e}(x, t)$ and a plastic $\bar{\kappa}^{p}(x, t)$ component [72]:

$$
\bar{\kappa}(x, t)=\bar{\kappa}^{e}(x, t)+\bar{\kappa}^{p}(x, t)
$$

- The moment curvature relation is written as

$$
M(x, t)=E I \bar{\kappa}^{e}(x, t)=E I\left(\bar{\kappa}(x, t)-\bar{\kappa}^{p}(x, t)\right)
$$

with $I$ the moment of inertia.

- The yield condition $\bar{\phi}$ is expressed as:

$$
\bar{\phi}(M, \bar{q})=|M|-\left(M_{c}-\bar{q}\right) \leq 0
$$

where $M$ the current bending moment, $M_{c}$ the elastic limit (flow stress), $M_{y}$ the value where the second slope change occurs and $M_{u}$ the failure (ultimate) bending moment of the moment curvature continuous model

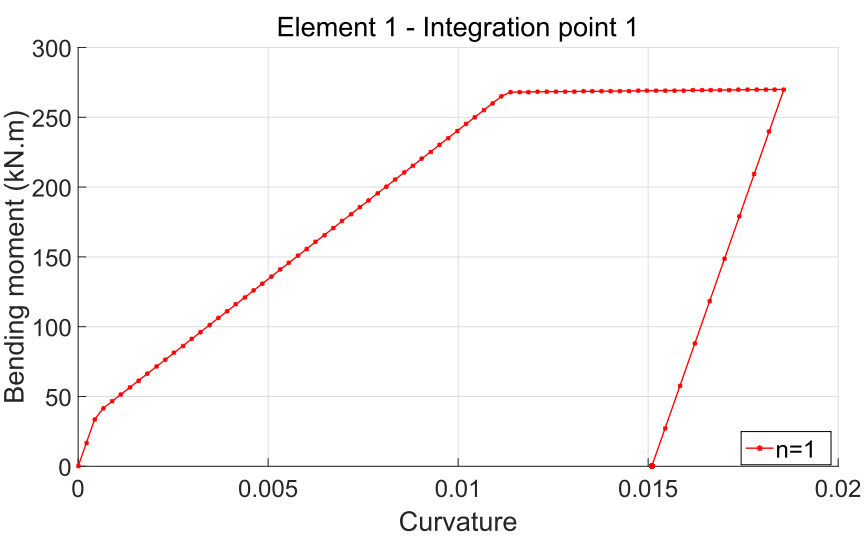

Fig. 12. Cantilever beam structure subjected to a rotation - 1 FCQ element Material behaviour outside the discontinuity.

(Fig. 4a). $\bar{q}$ is the stress-like internal hardening variable defined as:

$\bar{q}= \begin{cases}-H_{1} \bar{\xi} & \text { for } \bar{\xi} \leq \bar{\xi}_{y} \\ -\left(M_{y}-M_{c}\right)\left(1-\frac{H_{2}}{H_{1}}\right)-H_{2} \bar{\xi} & \text { for } \bar{\xi}>\bar{\xi}_{y}\end{cases}$

with $H_{1}$ and $H_{2}$ the two plastic moduli, $\bar{\xi} \geq 0$ the strain-like internal hardening variable and $\bar{\xi}_{y}=\frac{M_{y}-M_{c}}{H_{1}}$ (Fig. 4b).

- An associated plasticity is considered, the flow rule and the isotropic hardening law become (the principle of maximum plastic dissipation is as usual adopted to determine the evolution of the internal variables):

$\dot{\bar{\kappa}}^{p}=\dot{\bar{\gamma}} \operatorname{sign}(M) \quad \dot{\bar{\xi}}=\dot{\bar{\gamma}}$

where $\bar{\gamma}$ is the slip rate and $\operatorname{sign}(\bullet)$ defines the sign of the variable $(\bullet)$.

- The Kuhn-Tucker complementarity conditions are [72]:

$$
\dot{\bar{\gamma}} \geq 0 \quad \bar{\phi} \leq 0 \quad \dot{\bar{\gamma}} \bar{\phi}=0
$$

- The consistency condition is [72]:

$$
\dot{\bar{\gamma}} \dot{\bar{\phi}}=0
$$

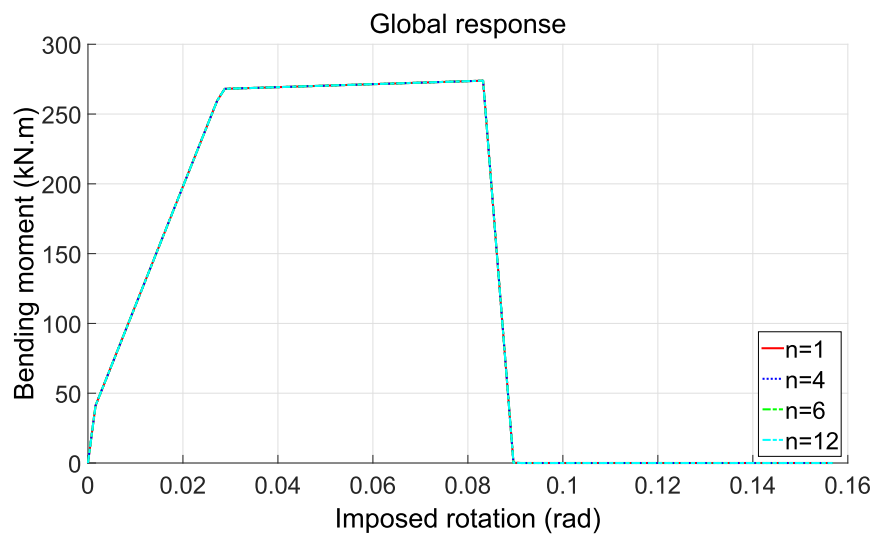

Fig. 11. Cantilever beam structure subjected to a rotation - Global response (bending moment - imposed rotation), presence of a default in the first FCQ element $\left(M_{u}^{e=1} 272 \mathrm{kN} \mathrm{m}\right.$ instead of $\left.274 \mathrm{kN} \mathrm{m}\right)$.

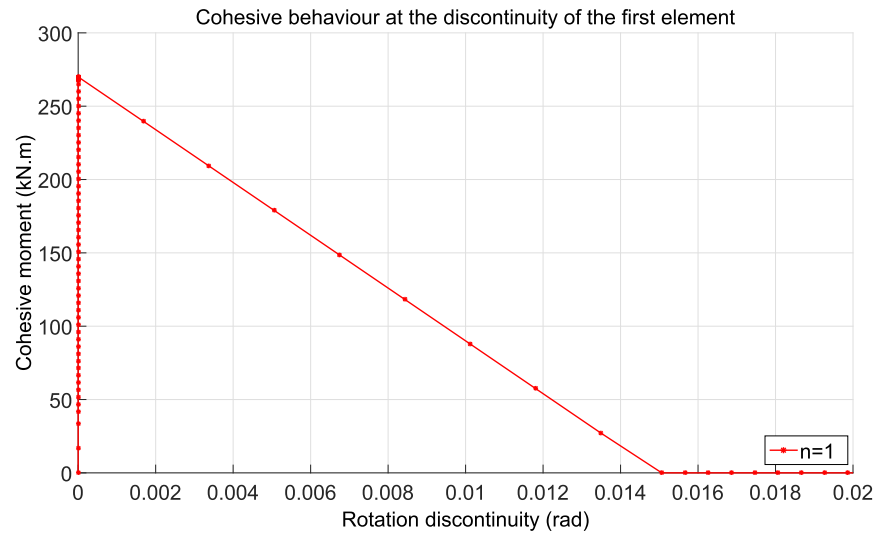

Fig. 13. Cantilever beam structure subjected to a rotation - 1 FCQ element Material behaviour at the discontinuity. 


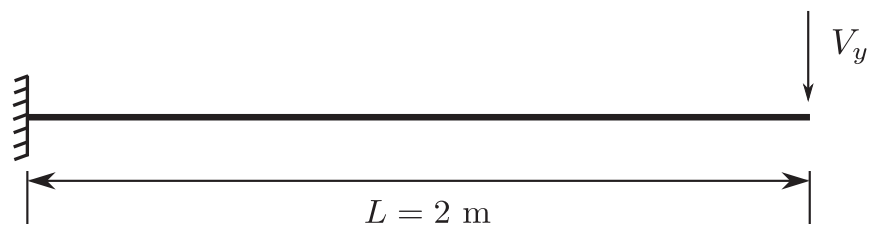

Fig. 14. Cantilever beam structure subjected to a transversal displacement.

For a plastic step, the slip rate $\dot{\bar{\gamma}}$ must be positive (not zero), so the rate of the yield surface $\dot{\bar{\phi}}$ must be zero. This allows determining $\dot{\bar{\gamma}}$ as follows:

$\dot{\gamma}= \begin{cases}\frac{E I \dot{\bar{\kappa}}}{E I+H_{1}} & \text { if } \bar{\xi} \leq \overline{\xi_{y}} \\ \frac{E I \dot{\bar{\kappa}}}{E I+H_{2}} & \text { otherwise }\end{cases}$

- Substitution of the last equation (55) in equation (49) finally gives:

$\dot{M}= \begin{cases}E I \dot{\bar{\kappa}} & \text { if } \dot{\gamma}=0 \\ E I\left(1-\frac{E I}{E I+H_{1}}\right) \dot{\bar{\kappa}} & \text { if } \bar{\xi} \leq \overline{\xi_{y}} \\ E I\left(1-\frac{E I}{E I+H_{2}}\right) \dot{\bar{\kappa}} & \text { otherwise }\end{cases}$

\subsection{Cohesive model}

The works of [73] and [74] are the first dealing with the development of cohesive models, models that link a stress to a displacement jump. They constitute an intermediate approach between continuum and fracture mechanics and are considered as an extension of the Griffith's theory [75] since they allow modeling crack initiation and evolution (see for example [43,76]). A significant improvement of this approach was proposed in Ref. [77] by introducing the concept of fracture energy and the critical stress beyond which the crack is supposed to propagate. The transition from the continuous to the cohesive model occurs when a criterion, often based on the stress state, is verified. As soon as the stress value (in our case the bending moment) at a given location exceeds the failure moment $M_{u}$, the discontinuity and its cohesive behaviour law are activated. Several cohesive laws exist in the literature according to the type of the material and the loading envisaged, $[43,76]$. Since only monotonic loadings are considered in this paper, a linear cohesive law is adopted hereafter for simplicity.

In the following, the adopted cohesive model links the cohesive bending moment $C_{m}$ to a rotation jump $\overline{\bar{\Theta}}_{e}$ with a linear law (Fig. 5). The cohesive relation is written as:

$C_{m}=S \overline{\bar{\Theta}}_{e}+M_{u}$

with $S$ (negative value) the softening modulus. $M_{u}$ and $S$ are chosen from experimental studies $[78,79]$ or multiscale identification calculations $[59,60]$.

The discontinuity represents a cohesive zone of zero thickness positionned at $x_{d}$ (where the failure moment $M_{u}$ is reached). As for the case of the continuous model, discontinuity activation is provided with a failure condition that takes the following form:

$\overline{\bar{\phi}}\left(C_{m}, \overline{\bar{\Theta}}_{e}\right)=\left|C_{m}\right|-\left(M_{u}+S \overline{\bar{\Theta}}_{e}\right) \leq 0$
After the activation of a discontinuity within the beam finite element, it is assumed that outside the material unloads elastically (the dissipation phenomena are supposed negligible outside the discontinuity, they only develop inside the cohesive zone [80]). Among the internal variables that verify the failure criterion, those that maximize the cohesive dissipation are selected. As for the continuous elastoplastic formulation, a minimization problem of free energy under constraint is solved and the discontinuity evolution equation becomes:

$\dot{\bar{\Theta}}_{e}=\dot{\bar{\gamma}} \operatorname{sign}\left(C_{m}\right)$

The Kuhn-Tucker complementarity conditions are:

$\dot{\bar{\gamma}} \geq 0, \quad \overline{\bar{\phi}} \leq 0, \quad \dot{\bar{\gamma}} \overline{\bar{\phi}}=0$

and the consistency condition:

$\dot{\bar{\gamma}} \dot{\bar{\phi}}=0$

When a discontinuity is active, the multiplier $\dot{\bar{\gamma}}$ is strictly positive. Thus, in order to verify the consistency condition $\dot{\bar{\phi}}=$ 0 . Using the equations (58) and (59) this finally results to:

$$
\left.\begin{array}{c}
\dot{\overline{\bar{\phi}}}=\left|\dot{C}_{m}\right|-S \dot{\overline{\bar{\Theta}}}_{e} \\
\Rightarrow 0=\left|\dot{C}_{m}\right|-S \dot{\bar{\Theta}}_{e} \\
\Rightarrow 0=\left|\dot{C}_{m}\right|-S \dot{\bar{\gamma}} \operatorname{sign}\left(C_{m}\right)
\end{array}\right\} \Longrightarrow \dot{\bar{\gamma}}=\frac{1}{S}\left|\dot{C}_{m}\right|=\frac{1}{S} \dot{C}_{m} \operatorname{sign}\left(C_{m}\right)
$$

\section{Numerical implementation}

After the presentation of the variational formulation, the enhanced generalized Timoshenko beam formulation and the continuous and cohesive laws, the computational implementation is detailed hereafter.

\subsection{Linearization of equilibrium equations}

The two equilibrium equation (33), repeated hereafter as equation (63), must be checked at each calculation step.

$$
\begin{aligned}
& \mathbf{F}_{\text {int }, B}^{s t r}(t)-\mathbf{F}_{\text {ext }}^{s t r}(t)=0 \\
& \forall e \in 1,2, \ldots, \overline{\bar{n}}: F_{\text {int }, G}^{e}(t)=0
\end{aligned}
$$

Equation (63) are generally non-linear. To solve them with the NewtonRaphson method they must be first linearized to obtain a system of linear coupled equations in a matrix form.

Suppose that all variables are known at $t$, we need to calculate them at $t+1$ (time step $t+1$ ). The residual $\mathbf{R}$ of the first (global) equation of (63) is defined as:

$$
\mathbf{R}\left(\mathbf{d}_{s t r}, \overline{\bar{\Theta}}_{1}, \overline{\bar{\Theta}}_{2}, \ldots, \overline{\bar{\Theta}}_{\overline{\bar{n}}}\right)=\mathbf{F}_{\text {int }, B}^{s t r}(t)-\mathbf{F}_{\text {ext }}^{s t r}(t)=\stackrel{A}{e=1}_{A}^{n}\left\{\mathbf{F}_{\text {int }, B}^{e}-\mathbf{F}_{\text {ext }}^{e}\right\}
$$

with

$$
\left\{\begin{array}{l}
\frac{\partial \mathbf{R}}{\partial \mathbf{d}_{s t r}}={ }_{e=1}^{n}\left(\frac{\partial \mathbf{F}_{\text {int, }, B}^{e}}{\partial \mathbf{d}_{e}}\right) \\
\frac{\partial \mathbf{R}}{\partial \overline{\bar{\Theta}}_{e}}=\frac{\partial \mathbf{F}_{\text {int }, B}^{e}}{\partial \overline{\bar{\Theta}}_{e}} \quad \forall e \in \overline{\bar{n}}
\end{array}\right.
$$

Table 1

Cantilever beam structure subjected to a transversal displacement - Properties of the generalized model.

\begin{tabular}{lllllll}
\hline$E I(\mathrm{kN} \mathrm{m})$ & $M_{c}(\mathrm{kN} \mathrm{m})$ & $M_{y}(\mathrm{kN} \mathrm{m})$ & $M_{u}(\mathrm{kN} \mathrm{m})$ & $H_{1}\left(\mathrm{kN} \mathrm{m}^{-2}\right)$ & $H_{2}\left(\mathrm{kN} \mathrm{m}^{-2}\right)$ & $S(\mathrm{kN} \mathrm{m})$ \\
\hline 4069000 & 37.9 & 275 & 302 & 50000 & 270 & -100000 \\
\hline
\end{tabular}




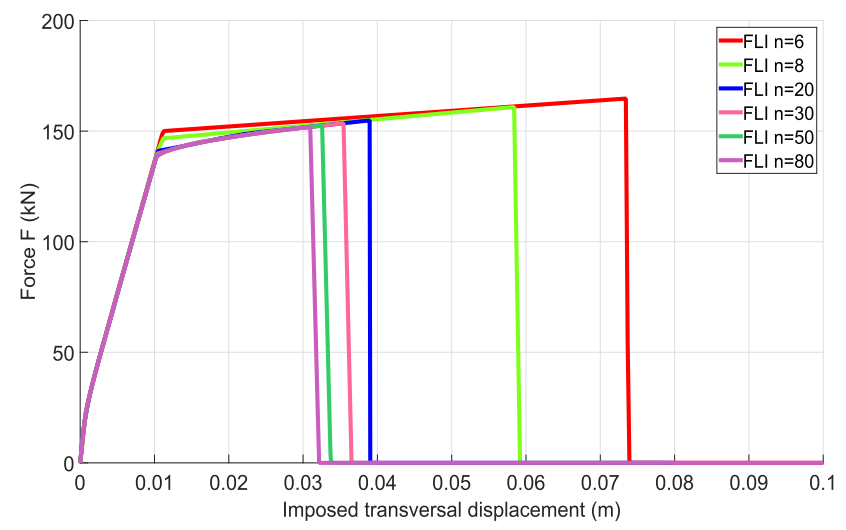

(a) FLI

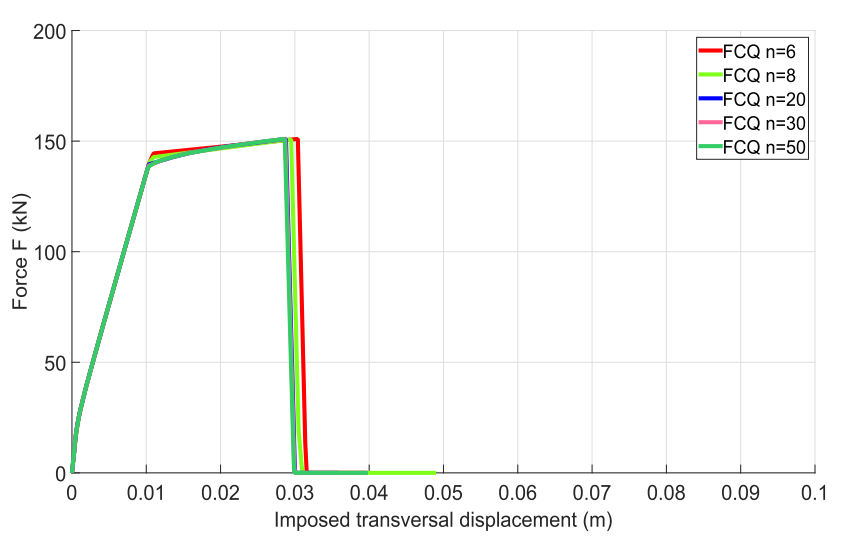

(b) FCQ

Fig. 15. Cantilever beam structure subjected to a transversal displacement - Global Response and mesh sensitivity.

$\mathbf{d}_{e}$ and $\overline{\bar{\Theta}}_{e}$ are respectively the vector of the continuous elementary nodal displacements and the elementary rotational discontinuity, As before, $n$ is the total number of elements, $\overline{\bar{n}} \leq n$ the number of elements where a discontinuity appears and $L$ the length of the element $e$. Linearization of equation (64) is done using an iterative procedure (the $k$ index is used for the iterations) to achieve structural equilibrium. For the time step $t+1$ we have:

$$
\begin{aligned}
& \left.\mathbf{R}\right|_{t+1} ^{(k+1)}=\left.\mathbf{R}\right|_{t+1} ^{(k)}+\left.\left.\frac{\partial \mathbf{R}}{\partial \mathbf{d}_{s t r}}\right|_{t+1} ^{(k)} \Delta \mathbf{d}_{s t r}\right|_{t+1} ^{(k+1)}+\sum_{e=1}^{\overline{\bar{n}}}\left\{\left.\left.\frac{\partial \mathbf{R}}{\partial \overline{\bar{\Theta}}_{e}}\right|_{t+1} ^{(k)} \Delta \overline{\bar{\Theta}}_{e}\right|_{t+1} ^{(k+1)}\right\}=0 \\
& \left.=\left.\mathbf{R}\right|_{t+1} ^{(k)}+\left.\stackrel{\mathrm{A}}{e=1}_{\mathbf{K}_{B B}}^{\left.\frac{\partial \mathbf{F}_{\mathrm{int}, B}^{e}}{\partial \mathbf{d}_{e}}\right|_{t+1} ^{(k)}} \cdot \Delta \mathbf{d}_{e}\right|_{t+1} ^{(k+1)}\right\} \\
& +\sum_{e=1}^{\overline{\bar{n}}}\{\left.\underbrace{\left.\frac{\partial \mathbf{F}_{\text {int }, B}^{e}}{\partial \overline{\bar{\Theta}}_{e}^{(k)}}\right|_{t+1}}_{\mathbf{K}_{B G}} \cdot \Delta \overline{\bar{\Theta}}_{e}\right|_{t+1} ^{(k+1)}\}=0
\end{aligned}
$$

Following the definition of $\mathbf{F}_{\text {int }, B}^{e}$ (see equation (30)), one can write:

$$
\begin{aligned}
\mathbf{K}_{B B}=\frac{\partial \mathbf{F}_{\text {int, },}^{e}}{\partial \mathbf{d}_{e}} & =\frac{\partial}{\partial \mathbf{d}_{e}}\left(\int_{L} \mathbf{B}(x)^{T} \mathbf{F}_{S}(x, t) d x\right)=\int_{L} \mathbf{B}(x)^{T} \frac{\partial \mathbf{F}_{S}}{\partial \mathbf{d}_{e}}(x, t) d x \\
& =\int_{L} \mathbf{B}(x)^{T} \frac{\partial \mathbf{F}_{S}}{\partial \widetilde{\varepsilon}_{S}}(x, t) \frac{\partial \widetilde{\varepsilon}_{S}}{\partial \mathbf{d}_{e}}(x) d x \\
& =\int_{L} \mathbf{B}(x)^{T} \frac{\partial \mathbf{F}_{S}}{\partial \widetilde{\varepsilon}_{S}}(x, t) \mathbf{B}(x) d x
\end{aligned}
$$

and

$$
\begin{aligned}
\mathbf{K}_{B G}=\frac{\partial \mathbf{F}_{\mathrm{int}, B}^{e}}{\partial \overline{\bar{\Theta}}_{e}} & =\frac{\partial}{\partial \overline{\bar{\Theta}}_{e}}\left(\int_{L} \mathbf{B}(x)^{T} \mathbf{F}_{S}(x, t) d x\right)=\int_{L} \mathbf{B}^{\kappa}(x)^{T} \frac{\partial M_{z}}{\partial \overline{\bar{\Theta}}_{e}}(x, t) d x \\
& =\int_{L} \mathbf{B}^{\kappa}(x)^{T} \frac{\partial M_{z}}{\partial \widetilde{\kappa}}(x, t) \frac{\partial \widetilde{\kappa}}{\partial \overline{\bar{\Theta}}_{e}}(x) d x \\
& =\int_{L} \mathbf{B}^{\kappa}(x)^{T} \frac{\partial M_{z}}{\partial \widetilde{\kappa}}(x, t) \bar{G}_{r}(x) d x
\end{aligned}
$$

Therefore, equation (66) can be reformulated in a matrix form (see also equation (64)):

$\underset{e=1}{\mathrm{~A}}\left\{\left[\begin{array}{ll}\mathbf{K}_{B B} & \mathbf{K}_{B G}\end{array}\right]_{t+1}^{(k)}\left[\begin{array}{c}\Delta \mathbf{d}_{e} \\ \Delta \overline{\bar{\Theta}}_{e}\end{array}\right]_{t+1}^{(k+1)}\right\}=-\left.\mathbf{R}\right|_{t+1} ^{(k)}=-\stackrel{\mathrm{A}}{e=1}^{n}\left\{\left.\left(\mathbf{F}_{\mathrm{int}, B}^{e}-\mathbf{F}_{e x t}^{e}\right)\right|_{t+1} ^{(k)}\right\}$

In a similar way, the second (local) equation of (63) is linearized using an iterative procedure (the $l$ index is adopted for the local iterations). For the time step $t+1$ and considering a fixed displacement increment $\Delta \mathbf{d}_{e}$ at the element (local) level for the iteration $k$ we have:

$$
\begin{aligned}
\left.F_{\text {int }, G}^{e}\right|^{(l+1)} & =\left.F_{\text {int }, G}^{e}\right|^{(l)}+\left.\left.\frac{\partial F_{\text {int }, G}^{e}}{\partial \mathbf{d}_{e}}\right|^{(l)} \Delta \mathbf{d}_{e}\right|_{t+1} ^{(k)}+\left.\left.\frac{\partial F_{\text {int }, G}^{e}}{\partial \overline{\bar{\Theta}}_{e}}\right|^{(l)} \Delta \overline{\bar{\Theta}}_{e}\right|^{(l+1)}=0 \\
& =\left.F_{\text {int }, G}^{e}\right|^{(l)}+\left.\left.\mathbf{K}_{G B}\right|^{(l)} \Delta \mathbf{d}_{e}\right|_{t+1} ^{(k)}+\left.\left.K_{G G}\right|^{(l)} \Delta \overline{\bar{\Theta}}_{e}\right|^{(l+1)}=0
\end{aligned}
$$

According to $F_{\text {int, } G}^{e}$ in equation (34), one can thus write:

$$
\begin{aligned}
\mathbf{K}_{G B}=\frac{\partial F_{\text {int }, G}^{e}}{\partial \mathbf{d}_{e}} & =\frac{\partial}{\partial \mathbf{d}_{e}}\left(\int_{L} \bar{G}_{v}(x)^{T} M_{z}(x, t) d x\right)=\int_{L} \bar{G}_{v}(x)^{T} \frac{\partial M_{z}}{\partial \mathbf{d}_{e}}(x, t) d x \\
& =\int_{L} \bar{G}_{v}(x)^{T} \frac{\partial M_{z}}{\partial \widetilde{\kappa}}(x, t) \frac{\partial \widetilde{\kappa}}{\partial \mathbf{d}_{e}}(x) d x \\
& =\int_{L} \bar{G}_{v}(x)^{T} \frac{\partial M_{z}}{\partial \widetilde{\kappa}}(x, t) \mathbf{B}^{\kappa}(x) d x
\end{aligned}
$$

and

$$
\begin{aligned}
K_{G G}=\frac{\partial F_{\text {int }, G}^{e}}{\partial \overline{\bar{\Theta}}_{e}} & =\frac{\partial}{\partial \overline{\bar{\Theta}}_{e}}\left(\int_{L} \bar{G}_{v}(x)^{T} M_{z}(x, t) d x\right)+\frac{\partial C_{m}(t)}{\partial \overline{\bar{\Theta}}_{e}} \\
& =\int_{L} \bar{G}_{v}(x)^{T} \frac{\partial M_{z}}{\partial \overline{\bar{\Theta}}_{e}}(x, t) d x+\frac{\partial C_{m}(t)}{\partial \overline{\bar{\Theta}}_{e}} \\
& =\int_{L} \bar{G}_{v}(x)^{T} \frac{\partial M_{z}}{\partial \widetilde{\kappa}}(x, t) \frac{\partial \widetilde{\kappa}}{\partial \overline{\bar{\Theta}}_{e}}(x) d x+\frac{\partial C_{m}(t)}{\partial \overline{\bar{\Theta}}_{e}} \\
& =\int_{L} \bar{G}_{v}(x)^{T} \frac{\partial M_{z}}{\partial \widetilde{\kappa}}(x, t) \bar{G}_{r}(x) d x+\frac{\partial C_{m}(t)}{\partial \overline{\bar{\Theta}}_{e}}
\end{aligned}
$$

with $\frac{\partial C_{m}(t)}{\partial \overline{\bar{\Theta}}_{e}}=S$ the softening modulus of the cohesive model (see equation (57)). Finally, assembling the local equation (70) of all the elements yields: 

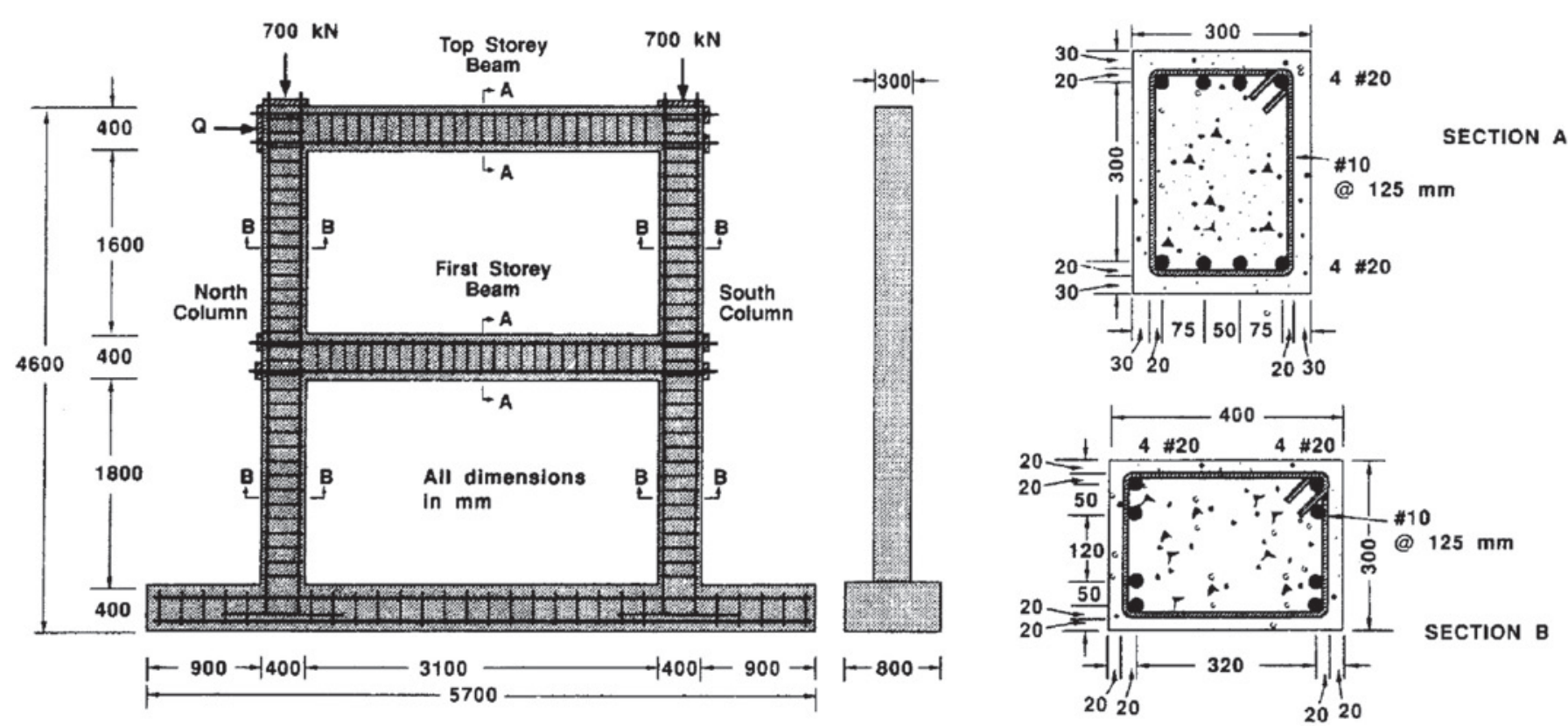

Fig. 16. Two-storey reinforced concrete frame - Geometry and reinforced concrete section details [86].

$\underset{e=1}{\mathrm{~A}}\left\{\left[\begin{array}{ll}\mathbf{K}_{G B} & K_{G G}\end{array}\right]_{t+1}^{(k)}\left[\begin{array}{c}\Delta \mathbf{d}_{e}^{(k)} \\ \Delta \overline{\bar{\Theta}}_{e}^{(l+1)}\end{array}\right]_{t+1}=-\left.F_{\mathrm{int}, G}^{e}\right|_{t+1} ^{(l)}\right\}$

Finally, equations (69) and (73) can be written in a single system as:

$\underset{e=1}{\mathrm{~A}}\left\{\left[\begin{array}{ll}\mathbf{K}_{B B} & \mathbf{K}_{B G} \\ \mathbf{K}_{G B} & K_{G G}\end{array}\right]_{t+1}^{(l)}\left[\begin{array}{c}\Delta \mathbf{d}_{e} \\ \Delta \overline{\bar{\Theta}}_{e}\end{array}\right]_{t+1}^{(k+1)}=\left[\begin{array}{c}-\left(\mathbf{F}_{\mathrm{int}, B}^{e}-\mathbf{F}_{e x t}^{e}\right) \\ -F_{\text {int }, G}^{e}\end{array}\right]_{t+1}^{(k)}\right\}$

The local variable $\Delta \overline{\bar{\Theta}}_{e}$ can be solved by static condensation at the element level [81]. The main advantage of the static condensation method is that the enhanced degrees of freedom are solved at the element (local) level and therefore the total number of degrees of freedom at the structural (global) level remains unchanged. After convergence of the internal discontinuities variables, we should have $\left.F_{\text {int }, G}^{e}\right|_{t+1}=0$. The system then becomes:

$\underset{e=1}{\mathrm{~A}}\left\{\left[\begin{array}{ll}\mathbf{K}_{B B} & \mathbf{K}_{B G} \\ \mathbf{K}_{G B} & K_{G G}\end{array}\right]_{t+1}^{(l)}\left[\begin{array}{c}\Delta \mathbf{d}_{e} \\ \overline{\bar{\Theta}}_{e}\end{array}\right]_{t+1}^{(k+1)}=\left[\begin{array}{c}-\left(\mathbf{F}_{\text {int }, B}^{e}-\mathbf{F}_{e x t}^{e}\right) \\ 0\end{array}\right]_{t+1}^{(k)}\right\}$

The second equation of the system gives:

$\left[\left.\mathbf{K}_{G B}\right|^{(l)} \Delta \mathbf{d}_{e}+\left.K_{G G}\right|^{(l)} \Delta \overline{\bar{\Theta}}_{e}\right]_{t+1}^{(k+1)}=0$

and therefore

$\left.\Delta \overline{\bar{\Theta}}_{e}\right|_{t+1} ^{(k+1)}=-\left.\left.\left.K_{G G}^{-1}\right|^{(l)} \mathbf{K}_{G B}\right|^{(l)} \Delta \mathbf{d}_{e}\right|_{t+1} ^{(k+1)}$

Table 2

Two-storey reinforced concrete frame - Generalized cross-sectional model parameters.

\begin{tabular}{ll}
\hline Variable & Description \\
\hline$M_{c}$ & Bending moment corresponding to the first crack in concrete fibers \\
$M_{y}$ & Bending moment beyond which steel fibers enter into plasticity \\
$M_{u}$ & Failure moment \\
$E I$ & Bending stiffness \\
$H_{1}$ & First hardening modulus (continuum model) \\
$H_{2}$ & Second hardening modulus (continuum model) \\
$S$ & Softening modulus (cohesive generalized model) \\
\hline
\end{tabular}

The use of the latter expression in the first equation of the system (75) yields:

$$
\begin{aligned}
& \left.\left.\mathbf{K}_{B B}\right|^{(l)} \Delta \mathbf{d}_{e}\right|_{t+1} ^{(k+1)}+\left.\left.\mathbf{K}_{B G}\right|^{(l)}\left(-\left.\left.K_{G G}^{-1}\right|^{(l)} \mathbf{K}_{G B}\right|^{(l)} \Delta \mathbf{d}_{e}\right)\right|_{t+1} ^{(k+1)} \\
& =-\left.\left(\mathbf{F}_{\text {int }, B}^{e}-\mathbf{F}_{e x t}^{e}\right)\right|_{t+1} ^{(k+1)}
\end{aligned}
$$

and consequently

$$
\begin{gathered}
\left.\underbrace{\left(\left.\mathbf{K}_{B B}\right|^{(l)}-\left.\left.\left.\mathbf{K}_{B G}\right|^{(l)} K_{G G}^{-1}\right|^{(l)} \mathbf{K}_{G B}\right|^{(l)}\right)}_{\left.\mathbf{K}_{\text {cond }}^{e}\right|^{(l)}} \Delta \mathbf{d}_{e}\right|_{t+1} \\
=-\left.\left(\mathbf{F}_{\text {int }, B}^{e}-\mathbf{F}_{e x t}^{e}\right)\right|_{t+1} ^{(k+1)}
\end{gathered}
$$

with $\mathbf{K}_{\text {cond }}^{e}$ the condensed stiffness matrix.

After the assembly of the element internal forces and stiffness matrices, the internal force vector of the structure $\mathbf{F}_{\text {int, } B}^{\text {str }}$ is introduced in the equation of the residual $\mathbf{R}_{t+1}^{(k+1)}$ (64). If $\mathbf{R}_{t+1}^{(k+1)}$ tends to zero, the next time step is triggered. Otherwise, another iteration $(k+2)$ is performed within the same time step $t+1$. A new displacement increment is calculated as follows:

$\delta \Delta \mathbf{d}_{e, t+1}^{(k+2)}=\left(\mathbf{R}_{t+1}^{(k+1)}\right)^{-1} \mathbf{K}_{t+1}^{(k+1)}$

with $\mathbf{K}_{t+1}^{(k+1)}$ the global stiffness matrix after the assembly of the elementary stiffness matrices. The new displacement increment of the step $t+1$ is:

$\Delta \mathbf{d}_{e, t+1}^{(k+2)}=\sum_{i=1}^{k+2} \delta \Delta \mathbf{d}_{e, t+1}^{(i)}$

The calculation procedure is repeated until both equilibrium equations (local and global) are verified. Once the equilibrium is reached, the internal variables and the structure displacement vector are updated as follows:

$\mathbf{d}_{e, t+1}^{\left(k^{\text {conv }}\right)}=\mathbf{d}_{e, t+1}^{(k)}+\Delta \mathbf{d}_{e, t+1}^{\left(k^{c o n v}\right)}$

with $k^{\text {conv }}$ the convergence iteration number. 
Table 3

Two-storey reinforced concrete frame - Generalized cross-sectional model parameters of columns and beams for the FLI [60], the Euler-Bernoulli [61] and the FCQ elements.

\begin{tabular}{|c|c|c|c|c|c|c|c|}
\hline \multirow[t]{2}{*}{ Parameter } & \multirow[t]{2}{*}{ Unit } & \multicolumn{3}{|l|}{ Columns } & \multicolumn{3}{|l|}{ Beams } \\
\hline & & FLI [60] & BER [61] & FCQ & FLI [60] & BER [61] & FCQ \\
\hline$E I$ & $\mathrm{kN} \mathrm{m}^{2}$ & 45760 & 45760 & 45760 & 45760 & 45760 & 45760 \\
\hline$M_{c}$ & $\mathrm{kN} \mathrm{m}$ & 114 & 100 & 100 & 33.1 & 30 & 30 \\
\hline$M_{y}$ & $\mathrm{kN} \mathrm{m}$ & 249 & 245 & 245 & 149 & 150 & 150 \\
\hline$M_{u}$ & $\mathrm{kN} \mathrm{m}$ & 260.2 & 265 & 295 & 160 & 170 & 185 \\
\hline$H_{1}$ & $\mathrm{kN} \mathrm{m}^{2}$ & 9020 & 12450 & 12450 & 8830 & 11190 & 11190 \\
\hline $\mathrm{H}_{2}$ & $\mathrm{kN} \mathrm{m}^{2}$ & 6.64 & 195 & 195 & 47.9 & 137 & 137 \\
\hline$S$ & $\mathrm{kN} \mathrm{m}$ & -5820 & -2410 & -2410 & -2540 & -1310 & -1310 \\
\hline
\end{tabular}

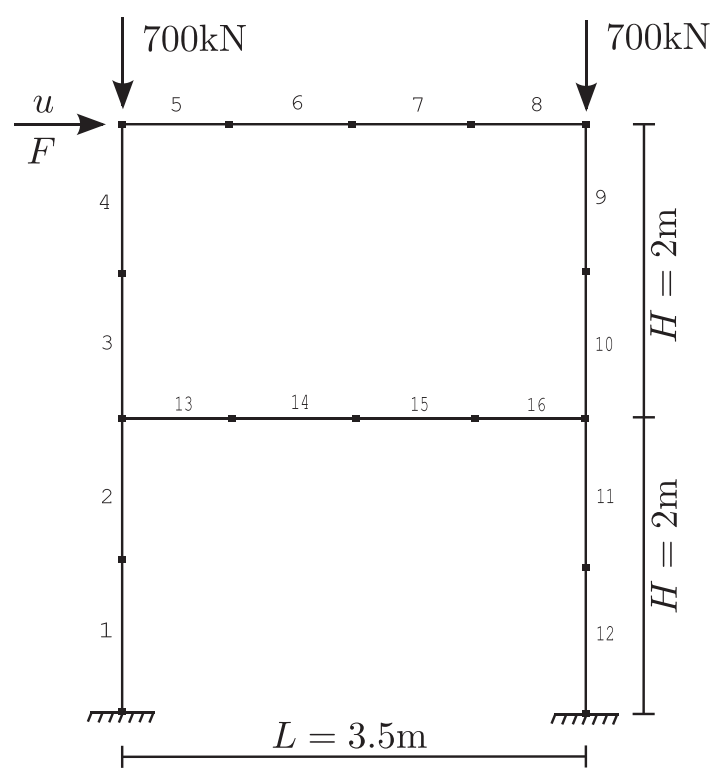

(a) Finite element mesh
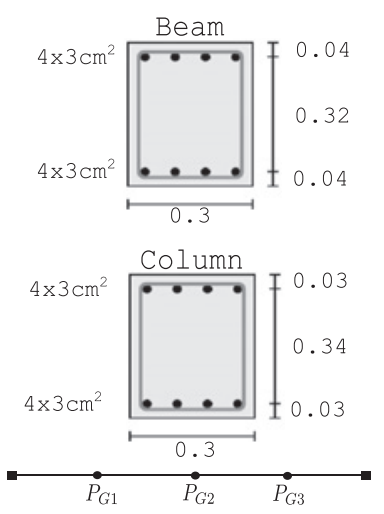

(b) Section details

Fig. 17. Two-storey reinforced concrete frame - Discretization, loading and section reinforcement details.

\subsection{Computation of the generalized constitutive model variables}

The evolution of the variables associated with the continuous $\left(\bar{\kappa}^{p}, \bar{\xi}\right)$ and the cohesive model $\left(\overline{\bar{\Theta}}_{e}\right)$ is calculated at each gauss point and for each iteration $k$. Since only monotonic loading is considered in this

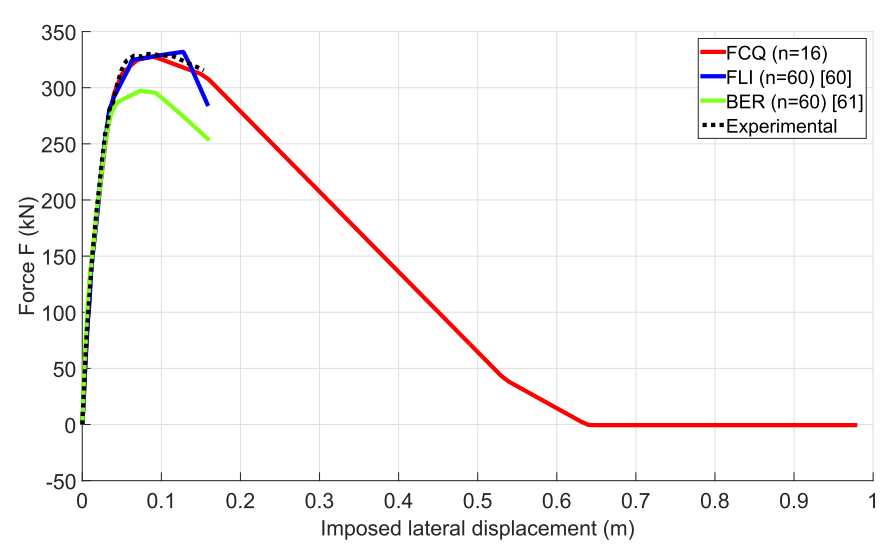

Fig. 18. Two-storey reinforced concrete frame - Numerical and experimental global results (force - horizontal displacement), FCQ, FLI and BER formulations. study, the two groups of variables cannot evolve simultaneously. Either the continuous model is active and only its internal variables evolve, either the cohesive model is active and outside the discontinuity the material undergoes an elastic unloading (see also section 5.2). The resolution procedure can therefore take two different paths (Path I and

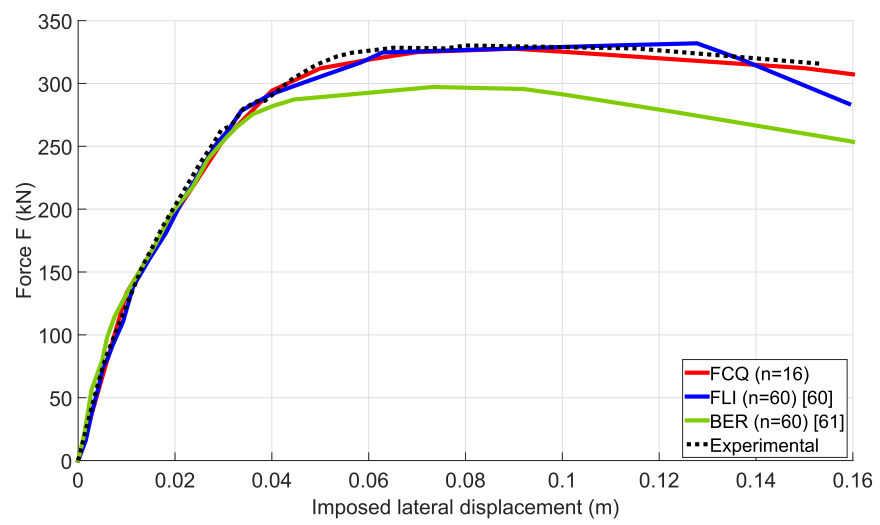

Fig. 19. Two-storey reinforced concrete frame - Numerical and experimental global results (force - horizontal displacement), FCQ, FLI and BER formulations (zoom between 0 and $0.16 \mathrm{~m}$ of imposed axial displacement). 


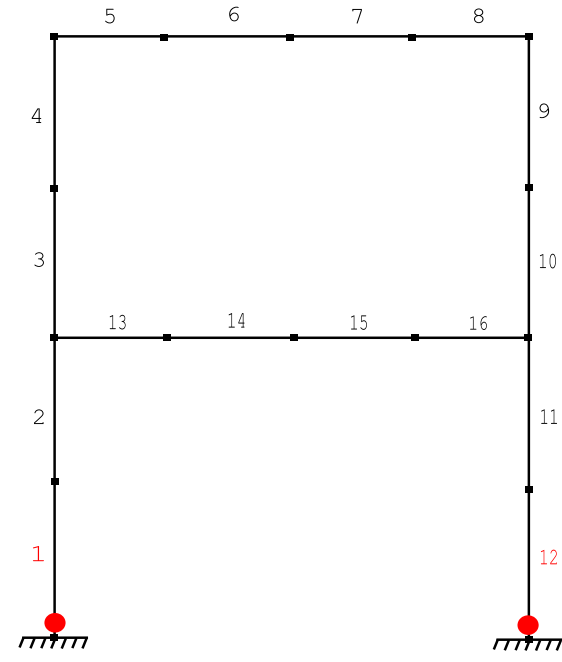

(a) $u(t=20)=0.095 \mathrm{~m}$

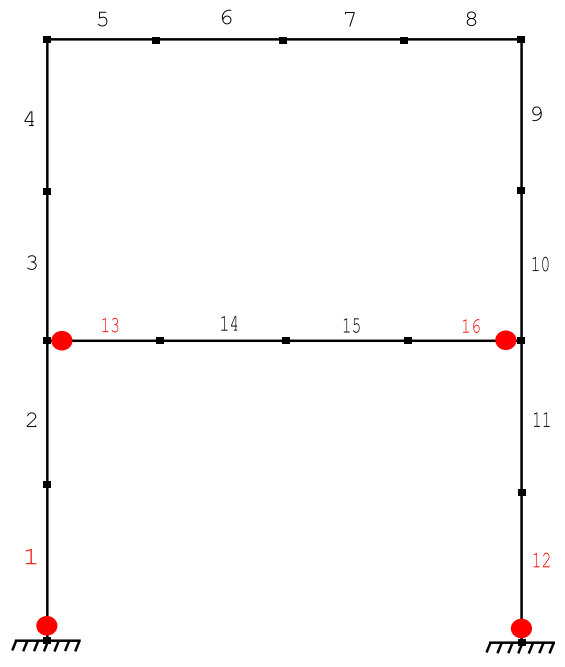

(b) $u(t=32)=0.155 \mathrm{~m}$

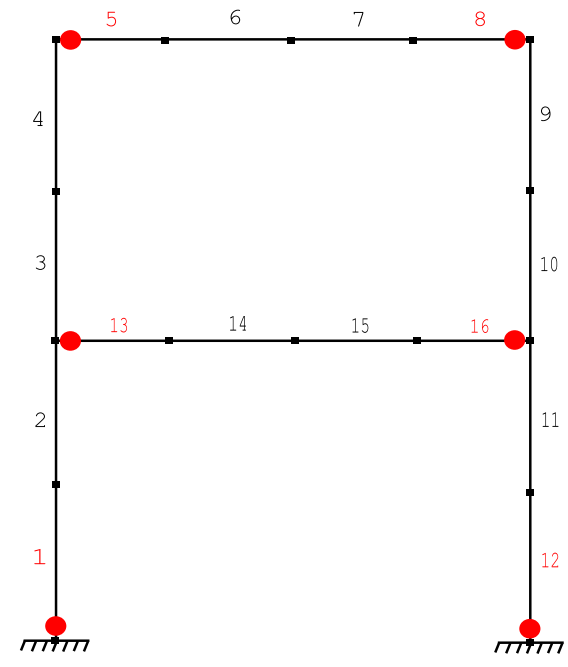

(c) $u(t=34)=0.165 \mathrm{~m}$

Fig. 20. Two-storey reinforced concrete frame - Progressive development of discontinuities, FCQ formulation.

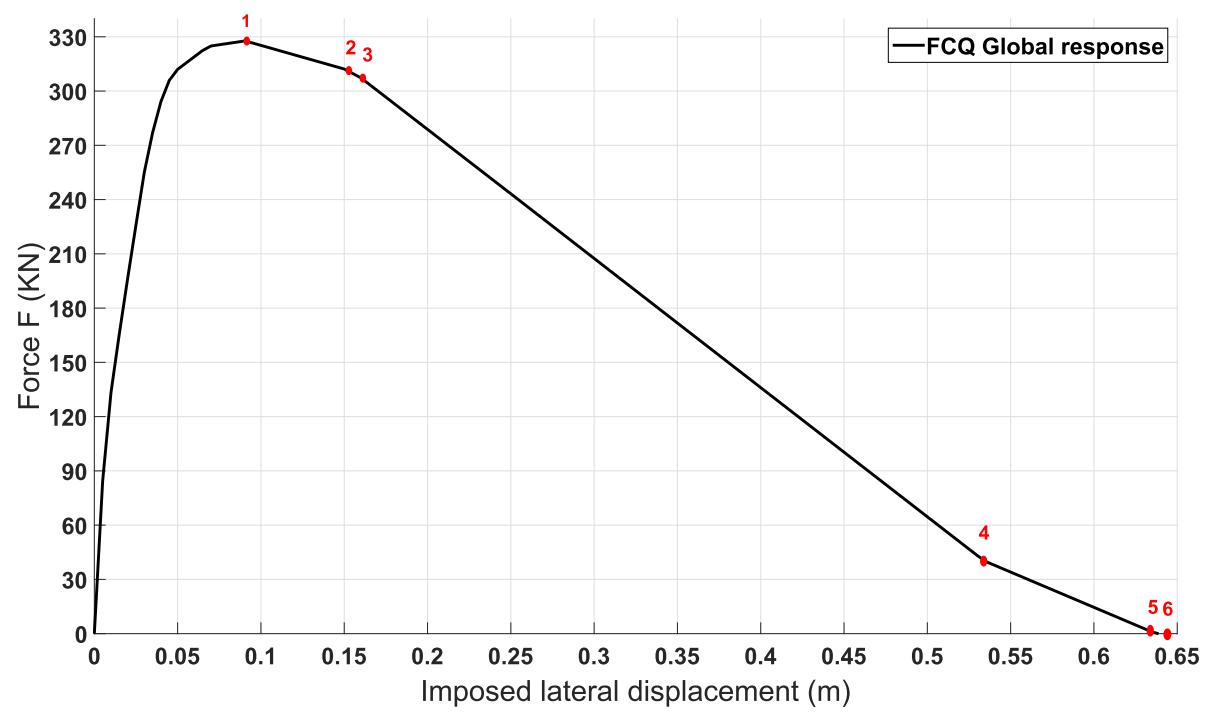

Fig. 21. Two-storey reinforced concrete frame - Progressive development of discontinuities on the global response curve, FCQ formulation.

Path II) depending on the discontinuity state.

Numerical implementation follows the Return-Mapping algorithms [82] and are based on the work of [58] and [83]. An implicit backward Euler integration scheme is adopted that takes the following general form $(v=1)$ :

$$
\begin{aligned}
& x_{n+1}=x_{n}+\overbrace{\Delta t \dot{x}_{n+v}}^{\Delta x} \\
& x_{n+v}=v x_{n+1}+(1-v) x_{n} ; v \in[0,1]
\end{aligned}
$$

\subsubsection{Plastic model variables}

The Path I (see Fig. 6) initial $(t)$ and final $(t+1)$ states are: Calculation is initiated assuming an elastic predictor:

$$
\begin{aligned}
\bar{\kappa}_{t+1}^{p, t r i a l} & =\bar{\kappa}_{t}^{p} \\
\bar{\xi}_{t+1}^{\text {trial }} & =\bar{\xi}_{t}
\end{aligned}
$$

The generalized moment and the hardening variable (stress-like) are calculated as:

$M_{z, t+1}^{\text {trial }}=E I\left[\bar{\kappa}\left(d_{e, t+1}^{(k-1)}, \overline{\bar{\Theta}}_{e, t+1}^{i,(k-1)}\right)-\bar{\kappa}_{t+1}^{p, t r i a l}\right]$

$\bar{q}_{t+1}^{\text {trial }}= \begin{cases}-H_{1} \bar{\xi}_{t+1}^{\text {trial }} & \text { if } \bar{\xi} \leq \bar{\xi}_{y} \\ -\left(M_{y}-M_{c}\right)\left(1-\frac{H_{2}}{H_{1}}\right)-H_{2} \bar{\xi}^{\text {trial }} & \text { otherwise }\end{cases}$

This makes possible to calculate $\bar{\phi}$ as:

$\bar{\phi}_{t+1}^{\text {trial }}=\left|M_{z, t+1}^{\text {trial }}\right|-\left(M_{c}-\bar{q}_{t+1}^{\text {trial }}\right) \leq 0$

If the last equation is verified then the step is elastic and the plastic and hardening variables do not change: 


$$
\begin{aligned}
\bar{\kappa}_{t+1}^{p} & =\bar{\kappa}_{t+1}^{p, t r i a l} \\
\bar{\xi}_{t+1} & =\bar{\xi}_{t+1}^{\text {trial }} \\
M_{z, t+1} & =M_{z, t+1}^{\text {trial }} \\
\bar{q}_{t+1} & =\bar{q}_{t+1}^{\text {trial }}
\end{aligned}
$$

If the stress state exceeds the elastic limit ( $\bar{\phi}^{\text {trial }}>0$ ), then the step is plastic and the variables are updated:

$$
\begin{aligned}
\bar{\kappa}_{t+1}^{p} & =\bar{\kappa}_{n}^{p}+\bar{\gamma}_{t+1} \operatorname{sign}\left(M_{z, t+1}^{\text {trial }}\right) \\
\bar{\xi}_{t+1} & =\bar{\xi}_{n}+\bar{\gamma}_{t+1} \\
M_{z, t+1} & =M_{z, t+1}^{\text {trial }}-\bar{\gamma}_{t+1} E I \operatorname{sign}\left(M_{z, t+1}\right)
\end{aligned}
$$

with $\bar{\gamma}_{t+1}$ the plastic multiplier calculated for $\bar{\phi}_{t+1}=0$.

The following property is used to resolve $\bar{\phi}_{t+1}=0$ :

$$
\operatorname{sign}\left(M_{z, t+1}\right)=\operatorname{sign}\left(M_{z, t+1}^{\text {trial }}\right)
$$

This can be seen from equation (89) as follows:

$$
\begin{aligned}
& \left|M_{z, t+1}\right| \operatorname{sign}\left(M_{z, t+1}\right)=\left|M_{z, t+1}^{t r i a l}\right| \operatorname{sign}\left(M_{z, t+1}^{\text {trial }}\right)-\bar{\gamma}_{t+1} E I \operatorname{sign}\left(M_{z, t+1}\right) \\
& \left(\left|M_{z, t+1}\right|+\bar{\gamma}_{t+1} E I\right) \operatorname{sign}\left(M_{z, t+1}\right)=\left|M_{z, t+1}^{\text {trial }}\right| \operatorname{sign}\left(M_{z, t+1}^{\text {trial }}\right)
\end{aligned}
$$

By definition $\bar{\gamma}_{t+1} \geq 0$ and therefore equation (91) provides:

$\operatorname{sign}\left(M_{z, t+1}\right)=\operatorname{sign}\left(M_{z, t+1}^{\text {trial }}\right)$

along with

$\left|M_{z, t+1}\right|=\left|M_{z, t+1}^{\text {trial }}\right|-\bar{\gamma}_{t+1} E I$

The updated stress-like hardening variable is:

$\bar{q}_{t+1}=\left\{\begin{array}{lll}\bar{q}_{t+1}^{\text {trial }}-H_{1} \bar{\gamma}_{t+1} & \text { if } \quad \bar{\xi}<\bar{\xi}_{t+1}<\bar{\xi}_{y} \\ \bar{q}_{t+1}^{\text {trial }}-\left(H_{2}-H_{1}\right)\left(\bar{\xi}_{t}-\bar{\xi}_{y}\right)-H_{2} \bar{\gamma}_{t+1} & \text { if } \bar{\xi}_{t} \leq \bar{\xi}_{y}, \bar{\xi}_{t+1}>\bar{\xi}_{y} \\ \bar{q}_{t+1}^{\text {trial }}-H_{2} \bar{\gamma}_{t+1} & \text { if } & \bar{\xi}_{t+1}>\bar{\xi}_{t}>\bar{\xi}_{y}\end{array}\right.$

Introducing equations (94) and (93) in the yield surface $\bar{\phi}_{t+1}$ provides the updated plastic multiplier $\bar{\gamma}_{t+1}$ as:

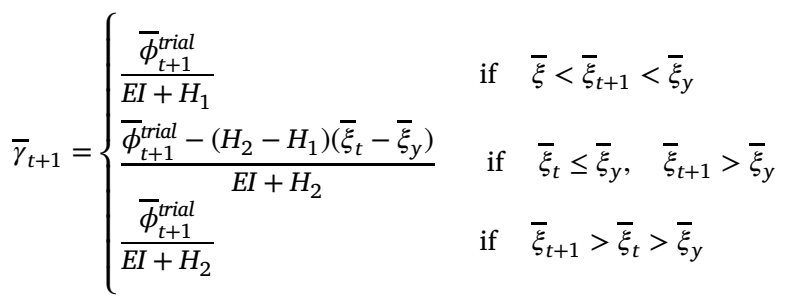

Finally, the discontinuity activation criterion within the element must be verified. The fracture surface $\overline{\bar{\phi}}\left(C_{t+1}, \overline{\bar{U}}_{t+1}\right)$ is first calculated and the cohesive force is found from equation (34):

$C_{m, t+1}=-\int_{0}^{L} \bar{G}_{v}(x) M_{z, t+1}(x) d x=-\frac{L}{2} \sum_{p g}^{n p g} \bar{G}_{v}\left(x_{p g}\right) M_{z, t+1}\left(x_{p g}\right) w\left(x_{p g}\right)$

with npg the number of integration points along the beam element and $w\left(x_{p g}\right)$ the associated integration weight of point $x_{p g}$. If $\overline{\bar{\phi}}\left(C_{m, t+1}, \overline{\bar{\Theta}}_{e, t+1}\right) \leq 0$, computation continues without activating the discontinuity. If $\overline{\bar{\phi}}\left(C_{m, t+1}, \overline{\bar{\Theta}}_{e, t+1}\right)>0$ the discontinuity is activated and all the updated values of the internal variables associated with the continuous model in this iteration are not considered. Calculation is re-started following the path II explained in the next paragraph.

\subsubsection{Cohesive model variables}

Path II (see Fig. 7) is followed when a discontinuity is active. In this case, only the variables associated with the cohesive model evolve. Outside the discontinuity the material undergoes elastic unloading and therefore the variables of the plastic model retain the values of the previous time step. The Path II initial $(t)$ and final $(t+1)$ states are:

The elastic predictor is written as:

$\overline{\bar{\Theta}}_{e, t+1}^{t r i a l}=\overline{\bar{\Theta}}_{e, t}$

The trial cohesive force $C_{t+1}^{t r i a l}=F_{t+1}\left(x_{d}\right)$ at the discontinuity level $x_{d}$ is determined using equation (34) as follows:

$C_{m, t+1}^{\text {trial }}=-\int_{L} \bar{G}_{v}(x) M_{z, t+1}^{t r i a l}(x) d x=-\frac{L}{2} \sum_{p g}^{n p g} \bar{G}_{v}\left(x_{p g}\right) M_{z, t+1}^{\text {trial }}\left(x_{p g}\right) w\left(x_{p g}\right)$

with

$M_{z, t+1}^{\text {trial }}\left(x_{p g}\right)=E I\left(x_{p g}\right)\left(B\left(x_{p g}\right) d_{e, t+1}^{(k-1)}+\bar{G}\left(x_{p g}\right) \overline{\bar{\Theta}}_{e, t+1}^{t r a l}-\bar{\kappa}_{t}^{p}\right)$

The failure sufrace $\overline{\bar{\phi}}_{t+1}^{\text {trial }}$ must be evaluated to check whether the discontinuity is activated or not:

$\overline{\bar{\phi}}_{t+1}^{\text {trial }}=\left|C_{m, t+1}^{\text {trial }}\right|-\left(M_{u}+S \overline{\bar{\Theta}}_{e, t+1}^{\text {trial }}\right) \leq 0$,

If the previous equation is verified then discontinuity is not active and the corresponding variables retain the predicted values:

$$
\begin{aligned}
& \overline{\bar{\Theta}}_{e, t+1}=\overline{\bar{\Theta}}_{e, t+1}^{t r a l} \\
& C_{m, t+1}=C_{m, t+1}^{\text {trial }}
\end{aligned}
$$

If $\overline{\bar{\phi}}_{t+1}^{\text {trial }}>0$ then the discontinuity is active. The new admissible values of the variables associated with the cohesive model are calculated using the implicit backward Euler integration scheme:

$\overline{\bar{\Theta}}_{e, t+1}=\overline{\bar{\Theta}}_{e, t}+\Delta \overline{\bar{\gamma}} \operatorname{sign}\left(C_{m, t+1}^{\text {trial }}\right)$

The updated cohesive force at the step $t+1$ is written:

$$
\begin{aligned}
C_{m, t+1}= & -\int_{L} \bar{G}_{v}(x) M_{z, t+1}(x) d x \\
= & -\int_{L} \bar{G}_{v}(x) E I\left(B^{v}(x) d_{t+1}+\bar{G}_{r}(x) \overline{\bar{\Theta}}_{e, t+1}-\bar{\kappa}_{t}^{p}\right) d x \\
= & -\underbrace{-\int_{L} \bar{G}_{v}(x) E I\left(B^{v}(x) d_{t+1}+\bar{G}_{r}(x) \overline{\bar{\Theta}}_{e, t}-\bar{\kappa}_{t}^{p}\right) d x}_{C_{m, t+1}^{\text {tral }}} \\
& -\underbrace{\int_{L} \bar{G}_{v}(x) E I \bar{G}_{r}(x) d x}_{E_{m}} \Delta \overline{\bar{\Theta}}_{e}
\end{aligned}
$$

therefore,

$C_{m, t+1}=C_{m, t+1}^{\text {trial }}-E_{m} \Delta \overline{\bar{\gamma}} \operatorname{sign}\left(C_{m, t+1}^{\text {trial }}\right)$

The multiplier $\Delta \overline{\bar{\gamma}}$ is found as:

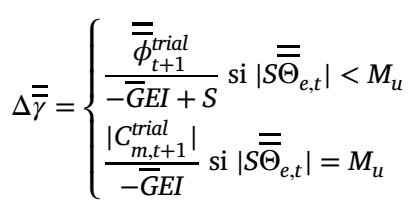

and finally, the tangent modulus is:

$\frac{d C_{m, t+1}}{d \overline{\bar{\Theta}}_{e}}=\left\{\begin{array}{l}\text { not defined if } \Delta \overline{\bar{\gamma}}=0 \\ S \text { if } \Delta \overline{\bar{\gamma}}>0 \text { and }\left|S \overline{\bar{\Theta}}_{e, t+1}\right|<M_{u} \\ 0 \text { if } \Delta \overline{\bar{\gamma}}>0 \text { and }\left|S \overline{\bar{\Theta}}_{e, t+1}\right|=M_{u}\end{array}\right.$ 
Once a discontinuity is activated for a given time step, it remains active for all subsequent time steps (monotonic loading is considered, crack closure is ignored). In the case of cyclic loading, reference should be made to reversible cohesive models that take into account crack closure. One way is to choose a cohesive model with linear closing as in Ref. [84]. More sophisticated cohesive models can also be based on damageplasticity models [43].

\section{Numerical applications}

All the previous developments are implemented in a Matlab Finite Element Toolbox that we are developing. The toolbox has various 2D finite elements as well as beam elements and allows for non-linear calculations using the classical Newton-Raphson scheme. It is based on the original work and software of [85].

\subsection{Cantilever beam structure submitted to a rotation}

In order to validate the previous developments, a cantilever beam structure of $L=2.5 \mathrm{~m}$ submitted to a rotation on its free end is considered in Fig. 8.

The enhanced FCQ Timoshenko beam is used for the spatial discretization and different numbers of elements are considered $(n=$ $1,4,6$ and 12). The generalized material behaviour is illustrated in Fig. 9. The discontinuity is supposed in the middle of each FCQ beam since the bending moment is constant.

For the first simulations presented hereafter, the FCQ elements have the same material characteristics. The global response (bending moment - imposed rotation) as a function of the number of elements is shown in Fig. 10. The bending moment takes its maximum value at the same time step for all discretizations. Mesh dependency however appears in the softening phase. Indeed, since the load is uniform throughout the beam, with increasing number of FCQ elements more discontinuities are activated and therefore the dissipated energy increases.

Note that the results of Fig. 10 is contrary to what we are used to see when there is localization. Usually, the more elements we have, the smaller the area is under the curve. Here it is the opposite because all discontinuities are activated as the same time as a result of the uniform load throughout the beam.

A default is then introduced in the first FCQ element (on the left) by decreasing its failure moment $\left(M_{u}^{e=1}: 272 \mathrm{kN} \mathrm{m}\right.$ instead of $\left.274 \mathrm{kN} \mathrm{m}\right)$. These new results are shown in Fig. 11. Imposing a default on the first element limits the number of activated discontinuities to a single discontinuity within the weakest (first) element. The mesh dependency vanishes and localization happens in a zero-thickness zone.

In order to understand what is happening at the local level, Figs. 12 and 13 illustrate respectively the material (continuous) behaviour at one integration point outside the discontinuity and at the discontinuity. Only one FCQ element is used for the discretization. The material behaviour outside the discontinuity evolves along the hardening path of Fig. 4 until the step where the discontinuity is activated. At this moment, the material outside the discontinuity discharges elastically (Fig. 12) to maintain the local balance with the cohesive model at the discontinuity (Fig. 13).

\subsection{Cantilever beam structure submitted to imposed transversal displacement}

The purpose of this example is to study the mesh sensitivity for a cantilever beam structure submitted to an imposed transversal displacement. The cantilever beam structure has a length $L=2 \mathrm{~m}$ and a rectangular cross-section. A transversal displacement $V_{y}$ is applied on its free end, Fig. 14.

The axial and transverse behaviour of the cross section is considered elastic (equation (47)), with $E A=4069000 \mathrm{kN}$ and $k G A=$
$1000000 \mathrm{kN}$. A continuous/cohesive coupled model is adopted for the moment - curvature behaviour (see section 5.1). Table 1 summarizes the properties of the generalized model.

The performance of twoTimoshenko enhanced formulations are presented in Fig. 15. The FLI formulation with linear interpolation functions [60] and the FCQ formulation considering high order interpolation functions. $n$ denotes the total number of finite element beams used to discretise the beam structure.

It is obvious that the FLI formulation requires a higher number of elements to converge to a stable global response, see Fig. 15a. On the other hand, the global response of the FCQ formulation is almost meshindependent, Fig. 15b.

\subsection{Two-storey reinforced concrete frame}

The purpose of this section is to validate the performance of the FCQ beam with embedded rotation discontinuity using the experimental results of a more complex structure, a two-storey reinforced concrete frame. Comparison is made with the results of the Timoshenko beam formulation with rotation discontinuity and linear independent shape functions for the rotations and the vertical displacements [49,60] (FLI) and an Euler-Bernoulli stress resultant beam finite element with rotation discontinuity (referred hereafter as BER) developed in Ref. [61] (a comparison of the FCQ and the FLI formulations without embedded discontinuities is presented in Ref. [51]).

The two-storey reinforced concrete frame was experimentally tested by Ref. [86] at scale 1 . Its geometrical and reinforced concrete section details are given in Fig. 16. Different sections are considered for the beams and the columns (see Fig. 16, sections A and B). In order to identify the parameters of the generalized models, the results of the multiscale analysis of [59] are adopted. The authors of [59] consider a multi-fiber beam discretization for the sections A and B (see Fig. 16) and were able to determine the parameters for the concrete and steel constitutive laws at the fiber level (and therefore the bending $E I$ and the axial elastic stiffnesses $E S$ ) but also to calibrate the hardening parameters $H_{1}, H_{2}$ and $S$ (see Table 2). The generalized cross-sectional model parameters are summarized in Table 3. The parameters for the FLI and BER formulations are the ones adopted in Refs. [60] and [61]. For FCQ element, the parameters of [61] are adopted with a small difference only for the $M_{u}$ value, on the basis of the experimental results of the two-storey frame [86].

In the following, normal and shear components are considered linear elastic as follows:

$F_{x}=E A \varepsilon_{x} \quad F_{y}=k G A \beta_{y}$

with $E A=3432000 \mathrm{kN}$ and $k G A=100000000 \mathrm{kN}$. However, an elasto-plastic model with an isotropic bi-linear hardening, as presented in Fig. 4, is considered to describe the generalized bending crosssectional behaviour. An increasing horizontal displacement is applied at the top of the structure. The comparison of the experimental and numerical results for the two enhanced Timoshenko (FCQ and FLI [60]) and Euler-Bernoulli beam formulations (BER [61]) is shown in Figs. 18 and 19.

The results of the FLI and BER enhanced formulations are taken respectively from Refs. [60] and [61]. In these simulations, the story height was divided into 16 and the span into 14 finite elements. The total number of elements for the discretisation of the entire structure is therefore $n=60$. For the enhanced FCQ beam presented in this paper, only 2 elements are considered for the discretization of the columns and 4 elements for the beams (see Fig. 17). The total number of elements for the discretisation of the entire structure is therefore $n=16$. Experimental results are only given up to a displacement of $0.15 \mathrm{~m}$. Beyond this value experimental results are not available [86]. Comparison of the FLI, BER and FCQ formulations is provided in Figs. 18 and 19. 
Fig. 18 clearly shows that the three enhanced formulations are able to correctly predict the elastic part. Significant differences are however observed in the plastic and the post peak response. The Timoshenkobased formulations (FLI and FCQ) match better the experimental results as they account for shear deformation. A smaller number of finite elements $(n=16)$ is needed for the FCQ formulation that provides the more realistic results, as it adoptes a higher order interpolation. This was also shown in a previous study (considering the FLI and FCQ formulations without enhancement) [51].

Fig. 20 illustrates the progressive development of discontinuities in the structure for the FCQ formulation. The first discontinuities appear on the frame supports at the lower ends of the columns (elements 1 and 12, Fig. 20a) after an imposed horizontal displacement of $0.095 \mathrm{~m}$ corresponding to a force of $F=326 \mathrm{kN}$. Then, two other discontinuities appear at both ends of the beams (13 and 16) of the first floor for an horizontal displacement of $0.155 \mathrm{~m}$ corresponding to a force of $F=310 \mathrm{kN}$ (20b). Finally, two last discontinuities occur at the ends of the upper beams ( 5 and 8 ) when the imposed horizontal displacement is equal to $0.165 \mathrm{~m}$ with a corresponding force of $F=304 \mathrm{kN}$ (20c).

Once the first plastic hinge is formed, a rigidity redistribution is performed at the structure level. The softening modulus $\frac{\partial C_{m}}{\partial \overline{\bar{\Theta}}_{e}}=S$ at the discontinuity level becomes zero. Therefore, the rigidity expression $K_{G G}$ in equation (72) is limited to the first term. After the formation of several plastic hinges total failure occurs. The global force response reaches therefore a zero value (see Fig. 18).

Fig. 21 illustrates the appearance of discontinuities on the overall structure response. Positions 1, 2 and 3 represent respectively the appearance of discontinuities at the lower ends of the columns (1 and 12) (Fig. 20a), at the ends of the intermediate beams (13 and 16) (Fig. 20b) and at the ends of the upper beams (5 et 8) (Fig. 20c). For this level of loading, discontinuities are not fully open, we can therefore considered them as plastic hinges. Positions 4, 5 and 6 correspond to completely open discontinuities. The sequences of their appearance is the same as before; first in the supports, then in the intermediate beams and finally in the upper beams.

\section{Conclusion}

A generalized enhanced Timoshenko beam formulation is proposed to simulate structural behaviour up to failure. The element uses higher order shape functions for the rotations and the vertical displacements improving thus its accuracy. A rotational discontinuity and the necessary enhancement functions are constructed following well-defined steps. The beam section is described by a generalized law that can be calibrated using experimental data or multi-scale finite element analysis (e.g. multi-fiber beams). The generalized bending material law (at the cross section level) has an elasto-plastic behaviour for the continuous part and a cohesive behaviour at the discontinuity level. The implementation procedures are detailed. Numerical examples considering a cantilever beam submitted to a rotation, to a transversal displacement and a two-storey reinforced concrete frame illustrate the capacity of the new beam formulation to regularize the results, to reproduce the development of plastic hinges and structural failure with relatively small computational cost (often from some seconds to minutes) compared to $2 \mathrm{D}$ or $3 \mathrm{D}$ classical finite elements (often from some minutes to hours).

In this paper, the shear response is considered elastic which is the starting point in several nonlinear analyses leading to the formulation of concentrated (localised) rotational plastic hinges. In order to reproduce shear failure however, the transversal displacement field has to be enhanced in a similar manner, see [Bui \& al. 2014]. Furthermore and in order to do dynamic calculations, a suitable cyclic cohesive model considering complete or partial closure is necessary. These issues will be addressed in a forthcoming work.

\section{Acknowledgments}

The authors would like to thank the PIA-SINAPS@ project (Sésisme et Installation Nucléaire: Améliorer et Pérenniser la Sureté), approved and funded by the National Agency of Research (ANR) following the RSNR 2012 call for projects on future investments post-Fukoshima. (SINAPS@ANR-11-RSNR-0022)

\section{References}

[1] I. Vardoulakis, M. Goldscheider, G. Gudehus, formation of shear bands in sand bodies as a bifurcation problem, Int. J. Numer. Anal. Meth. GeoMech. 2 (July 1977) (1978) 99-128, https://doi.org/10.1002/nag.1610020203.

[2] I. Vardoulakis, Rigid granular plasticity model and bifurcation in the triaxial test, Acta Mech. 49 (1-2) (1983) 57-79, https://doi.org/10.1007/BF01181755.

[3] Z.P. Bažant, Instability, ductility and size-effect in strain-softening concrete, J. Eng. Mech. Div. 1976 (April) (1976) 331-343.

[4] J. Mazars, A description of micro- and macroscale damage of concrete structures, Eng. Fract. Mech. 25 (5-6) (1986) 729-737, https://doi.org/10.1016/00137944(86)90036-6.

[5] W.R. Wawersik, W.F. Brace, Post-failure behavior of a granite and diabase, Rock Mechanics Felsmechanik Mécanique des Roches 3 (2) (1971) 61-85, https://doi. org/10.1007/BF01239627.

[6] H.E. Read, G.A. Hegemier, Strain Softening of rock, soil and concrete - a review article, Mech. Mater. 3 (1985) 271-294.

[7] J.R. Rice, The localization of plastic deformation. 14th International Congress on Theoratical and Applied Mechanics, 1976, pp. 207-220. doi:10.1.1.160.6740.

[8] G. Maugin, Nonlocal theories or gradient-type theories-a matter of convenience, Archiv of Mechanics, Archiwum Mechaniki Stosowanej 31 (1979) 15-26.

[9] G. Pijaudier-Cabot, Z.P. Bazant, Nonlocal damage theory, J. Eng. Mech. 113 (10) (1987) 1512-1533.

[10] E.C. Aifantis, On the microstructural origin of certain inelastic models, J. Eng. Mater. Technol. 106 (4) (1984) 326-330.

[11] R. Peerlings, R. De Borst, J. De Vree, Gradient enhanced damage for quasi-brittle materials, Int. J. Numer. Meth. Eng. 39 (1996) 3391-3403.

[12] R. de Borst, M. Geers, R. Peerlings, A. Benallal, Some remarks on gradient and nonlocal damage theories, in: J.-W.W.J. George, Z. Voyiadjis, J.-L. Chaboche (Eds.), Damage Mechanics in Engineering Materials, Vol. 46 of Studies in Applied Mechanics, Elsevier, 1998, pp. 223-236, https://doi.org/10.1016/S09225382(98)80044-5.

[13] N. Moës, C. Stolz, P.-E. Bernard, N. Chevaugeon, A level set based model for damage growth: the thick level set approach, Int. J. Numer. Meth. Eng. 86 (3) (2011) 358-380.

[14] F. Armero, D. Ehrlich, An analysis of strain localization and wave propagation in plastic models of beams at failure, Comput. Meth. Appl. Mech. Eng. 193 (30-32) (2004) 3129-3171, https://doi.org/10.1016/j.cma.2003.07.015.

[15] Z.P. Bažant, T.B. Belytschko, Wave propagation in a strain-softening bar: exact solution, J. Eng. Mech. 111 (3) (1985) 381-389.

[16] E. Cosserat, F. Cosserat, M. Brocato, K. Chatzis, Théorie des corps déformables, A. Hermann Paris, 1909.

[17] R. Toupin, Elastic materials with couple-stresses, Arch. Ration. Mech. Anal. 11 (1) (1962) 385-414.

[18] R. Mindlin, Micro-structure in linear elasticity, Arch. Ration. Mech. Anal. 16 (1) (1964) 51-78.

[19] A. Eringen, Linear Theory of Micropolar Elasticity, Tech. rep., Purdue University Lafayette in school of aeronautics and astronautics, 1965.

[20] P. Germain, The method of virtual power in continuum mechanics. part 2: Microstructure, SIAM J. Appl. Math. 25 (3) (1973) 556-575.

[21] S. Forest, R. Sievert, Nonlinear microstrain theories, Int. J. Solid Struct. 43 (24) (2006) 7224-7245, https://doi.org/10.1016/j.ijsolstr.2006.05.012, size-dependent Mechanics of Materials.

[22] H. Muhlhaus, I. Vardoulakis, The thickness of shear bands in granular materials, Geotechnique 37 (3) (1987) 271-283.

[23] R. Chambon, D. Caillerie, N.E. Hassan, One-dimensional localisation studied with a second grade model, Eur. J. Mech. Solid. 17 (4) (1998) 637-656, https://doi. org/10.1016/S0997-7538(99)80026-6.

[24] R. Chambon, D. Caillerie, T. Matsuchima, Plastic continuum with microstructure, local second gradient theories for geomaterials: localization studies, Int. J. Solid Struct. 38 (46-47) (2001) 8503-8527, https://doi.org/10.1016/S00207683(01)00057-9.

[25] T. Matsushima, R. Chambon, D. Caillerie, Large strain finite element analysis of a local second gradient model: application to localization, Int. J. Numer. Meth. Eng 54 (4) (2002) 499-521.

[26] F. Collin, R. Chambon, R. Charlier, A finite element method for poro mechanical modelling of geotechnical problems using local second gradient models, Int. J. Numer. Meth. Eng. 65 (11) (2006) 1749-1772.

[27] P. Bésuelle, R. Chambon, F. Collin, Switching deformation modes in post-localization solutions with a quasibrittle material, J. Mech. Mater. Struct. 1 (7) (2006) 1115-1134.

[28] P. Kotronis, S. Al Holo, P. Bésuelle, R. Chambon, Shear softening and localization: modelling the evolution of the width of the shear zone, Acta Geotech. 3 (2) (2008) 85-97. 
[29] R. Fernandes, C. Chavant, R. Chambon, A simplified second gradient model for dilatant materials: theory and numerical implementation, Int. J. Solid Struct. 45 (20) (2008) 5289-5307, https://doi.org/10.1016/j.ijsolstr.2008.05.032.

[30] Y. Sieffert, S. Al Holo, R. Chambon, Loss of uniqueness of numerical solutions of the borehole problem modelled with enhanced media, Int. J. Solid Struct. 46 (17) (2009) 3173-3197.

[31] G. Jouan, P. Kotronis, F. Collin, Using a second gradient model to simulate the behaviour of concrete structural elements, Finite Elem. Anal. Des. 90 (2014) 50-60, https://doi.org/10.1016/j.finel.2014.06.002.

[32] M. Soufflet, G. Jouan, P. Kotronis, F. Collin, Using a penalty term to deal with spurious oscillations in second gradient finite elements, Int. J. Damage Mech., (under revision).

[33] A. Needleman, Material rate dependence and mesh sensitivity in localization problems, Comput. Meth. Appl. Mech. Eng. 67 (1) (1988) 69-85.

[34] B. Loret, J.H. Prevost, Dynamic strain localization in elasto-(visco-) plastic solids, part 1. general formulation and one-dimensional examples, Comput. Meth. Appl. Mech. Eng. 83 (3) (1990) 247-273.

[35] J.C. Simo, M.S. Rifai, A class of mixed assumed strain methods and the method of incompatible modes, Int. J. Numer. Meth. Eng. 29 (8) (1990) 1595-1638. http:// doi.wiley.com/10.1002/nme.1620290802.

[36] T.B. Belytschko, J. Fish, B.E. Engelmann, A finite element with embedded localization zones, Comput. Meth. Appl. Mech. Eng. 70 (1988) 59-89.

[37] N. Moës, J. Dolbow, T.B. Belytschko, A Finite Element method for crack growth without remeshing, Int. J. Numer. Meth. Eng. 150 (February) (1999) 131-150.

[38] T.B. Belytschko, T. Black, Elastic crack growth in Finite Elements with minimal remseshing, Int. J. Numer. Meth. Eng. 620 (July 1998) (1999) 601-620.

[39] J. Dolbow, N. Moës, T.B. Belytschko, An extended finite element method for modeling crack growth with frictional contact, Comput. Meth. Appl. Mech. Eng. 190 (2001) 6825-6846.

[40] T.B. Belytschko, N. Moës, S. Usui, C. Parimi, Arbitrary discontinuities in finite elements, Int. J. Numer. Meth. Eng. 50 (August 2000) (2001) 993-1013.

[41] M. Melenk, I. Babuskab, The partition of unity finite element method: basic theory and applications, Comput. Meth. Appl. Mech. Eng. 139 (1996) 289-314.

[42] G.N. Wells, L.J. Sluys, A new method for modelling cohesive cracks using finite elements, Int. J. Numer. Meth. Eng. 50 (12) (2001) 2667-2682, https://doi.org/ $10.1002 / \mathrm{nme} .143$.

[43] F. Cazes, Construction et implémentation de lois cohésives extrinsèques, Ph.D. thesis, L'Institut National des Sciences Appliquées de Lyon, 2010.

[44] E.N. Dvorkin, A.M. Cuitiño, G. Gioia, Finite elements with displacement interpolated embedded localization lines insensitive to mesh size and distortions, Int. J. Numer. Meth. Eng. 30 (September 1989) (1990) 541-564, https://doi.org/ 10.1002/nme.1620300311.

[45] J. Oliver, A.E. Huespe, P.J. Sánchez, A comparative study on finite elements for capturing strong discontinuities: E-FEM vs X-FEM, Comput. Meth. Appl. Mech. Eng. 195 (37-40) (2006) 4732-4752, https://doi.org/10.1016/j.cma.2005.09.020, http://linkinghub.elsevier.com/retrieve/pii/S0045782505005049.

[46] M. Jirásek, Numerical modeling of strong discontinuities, Revue française de génie civil 6 (6) (2002) 1133-1146.

[47] D. Caillerie, P. Kotronis, R. Cybulski, A Timoshenko finite element straight beam with internal degrees of freedom, Int. J. Numer. Anal. Meth. GeoMech. 39 (16) (2015) 1753-1773.

[48] Z. Friedman, J.B. Kosmatka, An improved two-node Timoshenko beam Finite Element, Comput. Struct. 47 (3) (1993) 473-481.

[49] P. Pegon, A Timoshenko Simple Beam Element in Castem 2000, Special Publication Nr. I.94.04, Applied Mechanics Unit, Institute for Safety Technology, Joint Research Centre, Commission of the European Communities, Italy, 1994. I-21020 ISPRA (VA).

[50] J. Guedes, P. Pegon, A.V. Pinto, A Fibre/Timoshenko Beam Element in Castem 2000, Special Publication Nr. I.94.31, Applied Mechanics Unit, Institute for Safety Technology, Joint Research Centre, Commission of the European Communities, Italy, 1994. I-21020 ISPRA (VA).

[51] I. Bitar, S. Grange, P. Kotronis, N. Benkemoun, A comparison of displacement-based timoshenko multi-fiber beams finite element formulations and elasto-plastic applications, Eur. J. Environ. Civil Eng. 22 (4) (2018) 464-490. https://doi.org/10.1080/19648189.2016.1210031.

[52] H. Rasheed, K. Dinno, An efficient nonlinear analysis of RC sections, Comput. Struct. 53 (3) (1994) 613-623, https://doi.org/10.1016/0045-7949(94)90105-8, http://linkinghub.elsevier.com/retrieve/pii/0045794994901058.

[53] G. Royer-Carfagni, Can a moment-curvature relationship describe the flexion of softening beams? Eur. J. Mech. Solid. 20 (2) (2001) 253-276, https://doi.org/10. 1016/S0997-7538(00)01128-1.

[54] H.G. Kwak, S.P. Kim, Simplified monotonic moment-curvature relation considering fixed-end rotation and axial force effect, Eng. Struct. 32 (1) (2010) 69-79. https:// doi.org/10.1016/j.engstruct.2009.08.017.

[55] R. Nova, L. Montrasio, Settlements of shallow foundations on sand, Geotechnique 41 (2) (1991) 243-256.

[56] D. Ehrlich, F. Armero, Finite element methods for the analysis of softening plastic hinges in beams and frames, Comput. Mech. 35 (4) (2005) 237-264, https://doi. org/10.1007/s00466-004-0575-z.

[57] F. Armero, D. Ehrlich, Numerical modeling of softening hinges in thin Euler-Bernoulli beams, Comput. Struct. 84 (10-11) (2006) 641-656, https://doi. org/10.1016/j.compstruc.2005.11.010.

[58] J. Dujc, B. Brank, A. Ibrahimbegović, Multi-scale computational model for failure analysis of metal frames that includes softening and local buckling, Comput. Meth Appl. Mech. Eng. 199 (21-22) (2010) 1371-1385. https://doi.org/10.1016/j.cma. 2009.09.003.
[59] B.H. Pham, L. Davenne, D. Brancherie, A. Ibrahimbegović, Stress resultant mode for ultimate load design of reinforced-concrete frames: combined axial force and bending moment, Comput. Concr. 7 (4) (2010) 303-315, https://doi.org/10. 12989/cac. 2010.7.4.303, http://koreascience.or.kr/journal/view.jsp?kj=KJKHDQ $\& p y=2010 \& v n c=v 7 n 4 \& s p=303$.

[60] B.H. Pham, D. Brancherie, L. Davenne, A. Ibrahimbegović, Stress-resultant models for ultimate load design of reinforced concrete frames and multi-scale parameter estimates, Comput. Mech. 51 (3) (2012) 347-360, https://doi.org/10.1007/ s00466-012-0734-6, http://link.springer.com/10.1007/s00466-012-0734-6.

[61] M. Jukić, B. Brank, A. Ibrahimbegović, Embedded discontinuity finite element formulation for failure analysis of planar reinforced concrete beams and frames, Eng. Struct. 50 (2012) 115-125, https://doi.org/10.1016/j.engstruct.2012.07.028

[62] N.N. Bui, V.M. NGO, M. Nikolic, D. Brancherie, A. Ibrahimbegović, Enriched Timoshenko beam finite element for modeling bending and shear failure of reinforced concrete frames, Comput. Struct. 143 (2014) 9-18, https://doi.org/10. 1016/j.compstruc.2014.06.004, http://linkinghub.elsevier.com/retrieve/pii/ S0045794914001369.

[63] M. Jukić, B. Brank, A. Ibrahimbegović, Failure analysis of reinforced concrete frames by beam finite element that combines damage, plasticity and embedded discontinuity, Eng. Struct. 75 (2014) 507-527, https://doi.org/10.1016/j. engstruct.2014.06.017, http://linkinghub.elsevier.com/retrieve/pii/ S0141029614003733.

[64] O.C. Zienkiewicz, R.L. Taylor, Finite Element Method - the Basis, Butterworth-heinemann, Oxford, 2000.

[65] G. Casaux, A. Ibrahimbegović, On multi-fiber beam model with coupled axial and bending response, Eur. J. Comput. Mech. 20 (7-8) (2011) 411-426, https://doi. org/10.3166/ejcm.20.411-426.

[66] J. Oliver, Modelling strong discontinuities in solid mechanics via strain softening constitutive equations. Part 1: Fundamentals, Int. J. Numer. Meth. Eng. 39 (January) (1996) 3575-3600, https://doi.org/10.1002/(SICI)1097 0207(19961115)39:21\&iexcl;3601::AID-NME64\&iquest;3.0.CO;2-4.

[67] R.L. Taylor, J.C. Simo, O.C. Zienkiewicz, A.C.H. Chan, The patch test - a condition for assessing FEM convergence, Int. J. Numer. Meth. Eng. 22 (1) (1986) 39-62. http://doi.wiley.com/10.1002/nme.1620220105.

[68] B. M. Irons, A. Razzaque, Experience with the Patch Test for Convergence of Finite Elements, the Mathematical Foundations of the Finite Element Method with Applications to Partial Differential Equations 557.

[69] A. Ibrahimbegović, Mécanique non linéaire des solides déformables: Formulation théorique et résolution par éléments finis, Hermes science publ., 2006.

[70] G. Cowper, The shear coefficient in Timoshenko's beam theory, J. Appl. Mech. 33 (2) (1966) 335-340.

[71] B. Halphen, N. Q. Son, Sur les matériaux standards généralisés, J. Mec. 14 (1).

[72] R.I. Borja, Plasticity, Modeling \& Computation, Springer, 2013.

[73] D. Dugdale, Yielding of steel sheets containing slits, J. Mech. Phys. Solid. 8 (2) (1960) 100-104, https://doi.org/10.1016/0022-5096(60)90013-2, http://www. sciencedirect.com/science/article/pii/0022509660900132.

[74] G.I. Barenblatt, The mathematical theory of equilibrium cracks in brittle fracture, Adv. Appl. Mech. 7 (C) (1962) 55-129, https://doi.org/10.1016/S00652156(08)70121-2, arXiv:S0065-2156(08)70121-2.

[75] A.A. Griffith, The phenomena of rupture and flow in solids, Phil. Trans. Math. Phys. Eng. Sci. 221 (582-593) (1921) 163-198, https://doi.org/10.1098/rsta. 1921.0006, arXiv:arXiv:1011.1669v3.

[76] S. Cuvilliez, Passage d'un modèle d'endommagement continu régularisé à un modèle de fissuration cohésive dans le cadre de la rupture quasi-fragile, Tech. rep. EDF, 2013.

[77] A. Hillerborg, M. Modéer, P.E. Petersson, Analysis of crack formation and crack growth in concrete by means of fracture mechanics and finite elements, Cement Concr. Res. 6 (6) (1976) 773-781, https://doi.org/10.1016/0008-8846(76)900077.

[78] F.J. Vecchio, M.P. Collins, The modified compression-field theory for reinforced concrete elements subjected to shear, ACI J. Proceed. 83 (2) (1986) 219-231, https://doi.org/10.14359/10416, http://www.concrete.org/Publications/ ACIMaterialsJournal/ACIJournalSearch.aspx? $\mathrm{m}=$ details\&ID $=10416$.

[79] F.J. Vecchio, M.P. Collins, Predecting the response of reinforced concrete beams subjected to shear using modified compression field theory, ACI Struct. J. 85 (3) (1988) 258-268, https://doi.org/10.14359/2515.

[80] D. Brancherie, Modèles continus et discrets pour les problèmes de localisation et de rupture fragile et/ou ductile, Ph.D. thesis, École normale supérieure de Cachan, 2003.

[81] E.L. Wilson, The static condensation algorithm, Int. J. Numer. Meth. Eng. 8 (1) (1974) 198-203, https://doi.org/10.1002/nme.1620080115, http://doi.wiley. com/10.1002/nme.1620080115.

[82] J.C. Simo, T.J. Hughes, Computational Inelasticity, vol. 7, pringer Science \& Business Media, 1999, https://doi.org/10.1016/S0898-1221(99)90413-3.

[83] M. Jukić, Finite Elements for Modeling of Localized Failure in Reinforced Concrete, Ph.D. thesis, École normale supérieure de Cachan, 2013.

[84] F. Cazes, M. Coret, A. Combescure, A. Gravouil, A thermodynamic method for the construction of a cohesive law from a nonlocal damage model, Int. J. Solid Struct. 46 (6) (2009) 1476-1490.

[85] M. Bonnet, A. Frangi, Analyse des solides déformables par la méthode des éléments finis, Éditions de l’École Polytechnique, Palaiseau, France, 2006.

[86] F.J. Vecchio, M.B. Emara, Shear deformation in reinforced concrete frames, ACI Struct. J. 89 (No.1 Jan-Feb) (1992) 46-56. 